MEMORANDUM RM-4888-NASA MARCH 1966

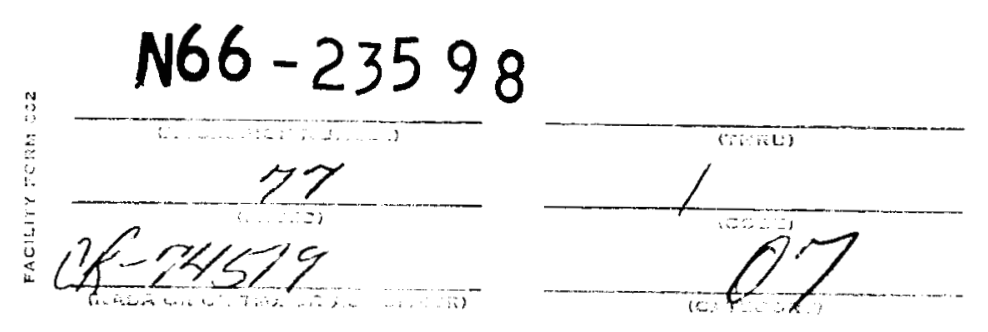

\title{
DISTORTION AND CROSSTALK OF LINEARLY FILTERED, ANGLE-MODULATED SIGNALS
}

E. Bedrosian

\begin{abstract}
GPO PRICE \$
\end{abstract}
CFSTI PRICE(S) $\$$

Hard Copv (HC)

$5<2$

Microfiche (MF)

.5

PREPARED FOR:

ff 653 July BE

NATIONAL AERONAUTICS AND SPACE ADMINISTRATION 
MEMORANDUM

RM-4888-NASA

MARCH 1966

DISTORTION AND CROSSTALK OF LINEARLY FILTERED, ANGLE-MODULATED SIGNALS

E. Bedrosian

This research is sponsored by the National Aeronautics and Space Administration under Contract No. NASr-21. This report does not necessarily represent the views of the National Aeronautics and Space Administration. 


\section{PREFACE}

This Memorandum was prepared as part of RAND's continuing study of Communications Satellite Technology for the National Aeronautics and Space Administration. It presents a new formulation which can be used to compute the distortion experienced by an FM signal as it passes throught the various circuits encountered in an FM receiver. The nature of the fundamental expansion used is such that it overcomes the major criticism of previous approaches to the problem.

The theoretical results of the Memorandum should add to the understanding of distortion and crosstalk in FM signals, and therefore constitute a significant contribution to communication theory and practice. The results can be used by engineers designing advanced communication systems to achieve optimum performance. 

signal, distortion and crosstalk components of the resulting signal are then presented graphically.

The importance of this example derives from the fact that the limited signal power available from communication satellites and space probes has stimulated the use of FM signals and frequency feedback receivers. Such receivers are constrained to the use of single-pole filters for reasons of stability and therefore incur considerable distortion and crosstalk in comparison with conventional FM receivers. The numerical results presented here will permit the selection of the proper filter bandwidth in order to attain the maximum threshold reduction while maintaining a specified level of crosstalk and distortion. 


\section{ACKNOWLEDGMENTS}

The author acknowledges with gratitude the many valuable discussions with Drs. W. Sollfrey and M. B. Marcus of The RAND Corporation and Professor I. S. Reed of the University of Southern California, Consultant to The RAND Corporation. Their suggestions and criticisms contributed greatly to the successful completion of this analysis. It should be noted that the use of the Taylor's series expansion described in Appendix B was suggested by Professor Reed. The programming of the IBM 7044 for the numerical computations of Section IV was performed by Mr. J. E. Rieber of The RAND Corporation.

Subsequent to the completion of the analysis reported in this Memorandum, it came to the author's attention that Dr. S. O. Rice of the Bell Telephone Laboratories had obtained substantially the same results for the third-order spectral components. As a result of the ensuing correspondence, a future joint publication of these and additional results is being considered. 


\section{CONTENTS}

PREFACE. ...........................

SUMMARY. ............................... v

ACKNOWLEDGMENTS. ............................ vii

LIST OF FIGURES..........................

LIST OF SYMBOLS............................. xiii

Section
\[ \text { I. INTRODUCTION. ............................. } 1 \]

II. INPUT-OUTPUT PHASE RELATIONSHIP................. 5

III. SPECTRAL ANALYSIS. .......................... 9

IV. NUMERICAL EXAMPLE--FM WITH UNIFORM BASEBAND

Appendix
\[ \text { EXPANSION OF OUTPUT PHASE } \ldots \ldots \ldots \ldots \ldots \ldots \ldots \ldots \ldots \ldots \ldots \ldots \]

B. TAYLOR'S SERIES EXPANSION.................... 37

C. SPECTRAL ANALYSIS .......................... 43

REFERENCES ............................... 55 


\section{LIST OF FIGURES}

1. Region of integration for crosstalk computation ....... 17

2. Output signal spectral density $\ldots \ldots \ldots \ldots \ldots \ldots \ldots \ldots \ldots$.

3. Output signal spectral density, decibels ........... 19

4. Output distortion spectral density .............. 20

5. Output distortion spectral density, decibels ........ 21

6. Output crosstalk spectral density $\ldots \ldots \ldots \ldots \ldots \ldots \ldots \ldots . \ldots . \ldots$

7. Output crosstalk spectral density, decibels .......... 24

8. Ratio of output signal-to-distortion spectral densities, decibels....................... 26

9. Ratio of output signal-to-crosstalk spectral densities, decibels ...................... 27

10. Output signal-to-distortion ratio, decibels.......... 29 


\section{LIST OF SYMBOLS}

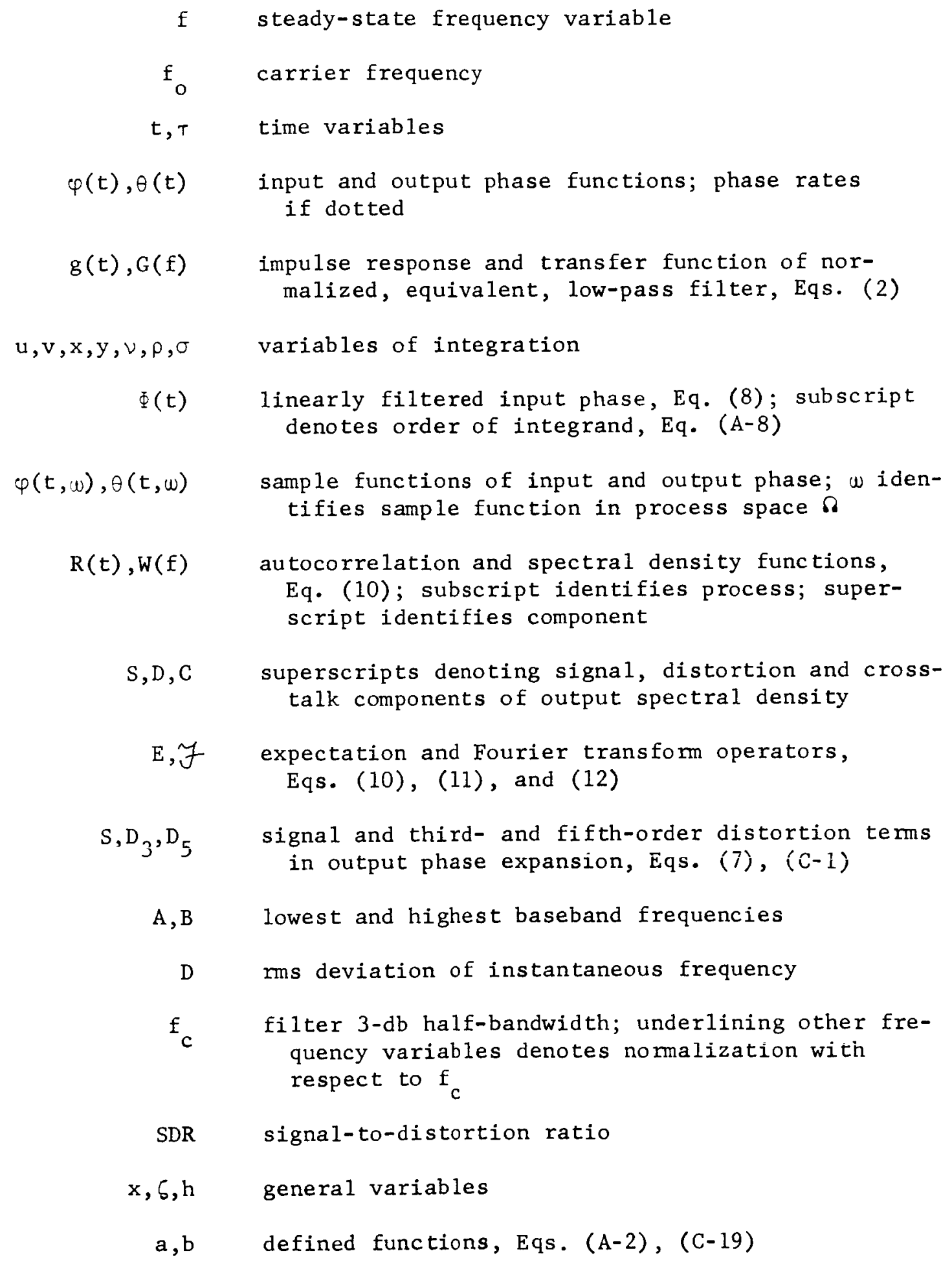




$$
\begin{aligned}
R & \text { series remainder, Eq. (A-9) } \\
\delta^{n} f(x ; h) & n^{\text {th }} \text { variation of } f(x) \text { with increment } h, E q \cdot(B-2) \\
R(\zeta), N(\zeta), D(\zeta) & \begin{array}{l}
\text { defined functions, } E q \cdot(B-9) ; \text { superscript denotes } \\
\text { order of derivative }
\end{array}
\end{aligned}
$$




\section{INTRODUCTION}

One of the most intriguing problems relating to the theory of angle (i.e., frequency or phase) modulation is that of obtaining a useful relationship between the properties of the input and output modulation when an angle-modulated signal is passed through a linear network. From the earliest work by Carson and Fry (1) in 1937 to the present, interest has persisted as others have extended results and added new approaches. These are reviewed in detail by Baghdady (2) and by panter, ${ }^{(3)}$ and it need be noted here only that the results have generally been presented either as infinite series involving Taylor's series expansions of both the modulating signal and the filter transfer function (Carson and Fry, ${ }^{(1)}$ Bloch, ${ }^{(4)}$ Van der Pol, (5) Stumpers, ${ }^{(6)}$ Clavier ${ }^{(7)}$ ), or as finite series involving the poles of the polynomial representation and but one derivative of the modulating signal (Hupert, $(8,9)$ Weiner and Leon $(10,11)$ ).

It is characteristic of all these expansions that they are in the form of a leading term called the quasi-stationary response and a remainder constituting a correction term. The quasi-stationaray response, a term introduced by Carson and Fry, is obtained by substituting the time-dependent, instantaneous frequency of the modulated signal for the steady-state frequency variable in the network transfer function. The quasi-stationary response is appealing intuitively since it is what might be expected if the frequency variations were made slowly enough. It has been useful analytically because the applications of interest are usually those in which the distortion is 
sufficiently small that the correction term can be neglected and the quasi-stationary response alone used in further analysis. As might be expected, considerable attention has been directed to questions of convergence and to obtaining bounds on the correction term (Baghdady, (2) Rowe, (12) Hess, (13) Weiner and Leon ${ }^{(11)}$ ).

Initial applications of these techniques were made using deterministic signals. Modulation using one ${ }^{(14-20)}$ or two ${ }^{(21)}$ sinusoids leads to a tractable analysis and yields results useful in determining harmonic distortion and the IF bandwidths required in FM receivers. (See Refs. 13, 22-24.) Swept frequency analyses $(25,26)$ have applications to spectrum analyzers, while responses to frequency steps and impulses $(5,27-30)$ are valuable in studies of impulsive interference, such as ignition noise, or in certain modulation techniques, such as frequency- or phase-shift keying.

More recently, analyses have been performed using stochastic modulating signals. These have been adapted to the square-law phase characteristic of the ionosphere by Shaft ${ }^{(31)}$ and Prosin ${ }^{(32)}$ and to the case of echo distortion by Bennett, Curtis and Rice. (33) Generalized analyses applicable to band-pass filters have been made by Rice, (34) Bosse, (35) Medhurst ${ }^{(36)}$ and Magnusson. (37) While these analyses have made significant contributions by considering more realistic modulating signals, they have been criticized because of their quasi-stationary nature (Bedrosian ${ }^{(38)}$ ) or their first-order basis (Enloe and Ruthroff ${ }^{(39)}$ ) inasmuch as terms of possibly significant contribution may have been discarded. 
Recently, the limited amount of signal power available in communication links from earth satellites and space probes has revived interest in the technique of FM with frequency feedback invented by Chaffee. (40) Considerations of stability require that a single-pole IF filter be used and Enloe ${ }^{(41)}$ shows that optimum threshold reduction requires that the IF filter have a $3-d b$ bandwidth comparable to the larger of the peak frequency deviation or the highest baseband frequency used (Ruthroff and Bodtmann, (42) Giger and Chaffee ${ }^{(43)}$ ). Thus, it has become all the more important that a satisfactory theoretical analys is be developed.

In contrast with a conventional FM receiver in which the IF amplifier can be designed to have an essentially linear phase characteristic and a uniform passband with steep skirts, an FM receiver employing feedback is forced to operate over an IF characteristic which is quite nonlinear. Although the feedback process itself helps reduce distortion, significant distortion and intermodulation or crosstalk can occur in practical situations and must be taken into account. In fact, at least one manufacturer of high-quality, multichannel systems finds the intermodulation sufficiently severe to warrant the use of threshold extension by frequency feedback only when the system falls below the conventional FM threshold. The analysis presented in this Memorandum does not proceed from the expansions customarily used. A signal with an arbitrary angle modulation is applied to a symmetrical, band-pass filter and an expression is obtained for the angle modulation on the output signal. This output phase is then expanded in a series in which the leading 
(first-order) term is recognized as the filtered but undistorted signal component of the output. The subsequent terms constitute the higher odd-order contributions to the output. A spectral analysis using stochastic modulation then derives the signal component of the output spectrum from the autocorrelation of the first-order term, the first-order distortion from the cross correlation of the firstand third-order terms, and the first-order crosstalk from the autocorrelation of the third-order term. The output signal, distortion and crosstalk spectra are computed numerically for the specific example of a signal frequency-modulated by a uniform spectrum of gaussian noise and passed through a single-pole filter.

Questions of convergence and of higher-order contributions due to neglecting the fifth- and higher-order terms in the expansion are considered. In general, it is argued that the first-order distortion and crosstalk computed herein constitute the principal distortion contributions to the output in the case of small distortion. Consequently, the results should find wide application in cases of practical interest. 


\section{INPUT-OUTPUT PHASE RELATIONSHIP}

Consider an angle-modulated signal of unit amplitude having a carrier frequency, $f_{o} c p s$, and a phase modulation, $\varphi(t)$, which, in complex form, can be written

$$
\exp i\left[2 \pi f_{0} t+\varphi(t)\right]
$$

Furthermore, let the properties of $\varphi(t)$ and its derivative, the phase rate $\dot{\varphi}(t)$, be such that the signal is narrow band, i.e., most of its energy is restricted to frequencies in the vicinity of $\mathrm{f}_{\mathrm{o}}$.

Take this angle-modulated signal as the input to a symmetrical, band-pass filter centered at $f_{o}$ and let the filter be sufficiently narrow that its transfer function can be well approximated by a symmetrical function of the linear off-center frequency difference, $f-f_{0}$, where $f$ is the steady-state frequency variable. It is then readily shown that the effect of band-pass filtering on the original signal is the same as the effect of filtering by the low-pass equivalent filter on the low-pass equivalent signal. That is, $f_{0}$ may simply be set equal to zero.

Let $G(f)$ and $g(t)$ denote, respectively, the nomalized steadystate transfer function and impulse response of the equivalent lowpass filcer. Then $G$ and $\tilde{g}$ are a Fourier pair

$$
g(t)=\int_{-\infty}^{\infty} d f G(f) e^{i 2 \pi f t}, \quad G(f)=\int_{0}^{\infty} d t g(t) e^{-i 2 \pi f t}
$$

where $g(t)$ is real and vanishes for $t<0$, and $G(f)$ is complex with an even real part and an odd imaginary part so that 


$$
G(f)=G^{*}(-f)
$$

where ${ }^{*}$ denotes the complex conjugate. The normalization chosen is

$$
\int_{0}^{\infty} d u g(u)=G(0)=1
$$

The filter output signal is given by the convolution of the input with the filter impulse response

$$
\int_{0}^{\infty} d u g(u) \exp [i \varphi(t-u)]
$$

and the output phase by the argument of Eq. (5)

$$
\theta(t)=\tan ^{-1} \frac{\int_{0}^{\infty} d u g(u) \sin \varphi(t-u)}{\int_{0}^{\infty} d u g(u) \cos \varphi(t-u)}
$$

Expanding as shown in Appendix A then leads to

$$
\begin{aligned}
\theta(t)= & \Phi(t)-\frac{1}{3 !} \int_{0}^{\infty} \operatorname{du} g(u)[\varphi(t-u)-\Phi(t)]^{3}+\frac{1}{5 !} \int_{0}^{\infty} \operatorname{dug} g(u)[\varphi(t-u)-\Phi(t)]^{5} \\
& -\frac{1}{12} \int_{0}^{\infty} \operatorname{du} g(u)[\varphi(t-u)-\Phi(t)]^{2} \int_{0}^{\infty} \operatorname{du} g(u)[\varphi(t-u)-\Phi(t)]^{3}+0\left(\varphi^{7}\right)
\end{aligned}
$$

where

$$
\Phi(t) \equiv \int_{0}^{\infty} d u g(u) \varphi(t-u)
$$


corresponds to the linearly filtered input phase and $0\left(\varphi^{7}\right)$ denotes terms of order 7 and higher in $\varphi(t)$. Necessary and sufficient conditions for Eq. (7) to converge for all $\varphi$ are that the Taylor's series for the input signal $\exp [i \varphi(t)]$ converge uniformly in the infinite interval on $t$, that the complex output signal given by Eq. (5) not vanish, and that

$$
\int_{0}^{\infty} \mathrm{du}|\mathrm{g}(\mathrm{u})|<\sqrt{2}
$$

The output phase rate, $\dot{\theta}(t)$, is then simply the time derivative of Eq. (7), providing the resulting series also converges uniformly.

It is difficult to state general conditions on the behavior at the input signal based on the foregoing and specific cases must be considered individually. However, the convergence condition of Eq. (9) does insure, in effect, that the filter impulse response and transfer function are sufficiently "well behaved." The condition is clearly satisfied by a first-order filter--inspection of impulse response plots shows it to be satisfied by Bessel filters of all orders and by low-order Butterworth and Tchebycheff filters as well.

The expansion given by Eq. (7) can also be obtained directly as a functional Taylor's series, ${ }^{(44)}$ as shown in Appendix B. Though the approach is more siraightorward, it does not appear possible to state conditions of convergence. At best, it confirms that the contributions of various orders are completely represented in the expansion.

The form of the input-output relationship given by Eq. (7) is particularly desirable in that the leading term clearly corresponds to the undistorted signal component of the output and is simply the 
linearly filtered input. The remaining terms then constitute the various higher odd orders of distortion and are interesting in that they highlight the difference between the delayed and filtered inputs, i.e., $\varphi(t-u)-\Phi(t)$, as the expansion parameter. This quantity is generally the least for that delay at which the impulse response is the greatest. For large delays, its growth is easily overcome by the eventual exponential decrease of all physically realizable impulse responses. Also, it is worth noting that the compactness of the expansion is itself of considerable value because it greatly facilitates the ensuing spectral analysis. 


\section{SPECTRAL ANALYSIS}

In communications systems analysis, the effect of the small nonlinearities encountered in signal processing is frequently better described in terms of the statistical properties of an ensemble of representative signals than by the explicit nature of a specific signal. Typical of the situations in which an angle-modulated signal can suffer significant distortion due to band-pass filtering is one in which the phase or instantaneous frequency of the carrier is made to vary in accordance with the amplitude of a multi-channel, frequencydivision-multiplex speech or data baseband signal. For many purposes, such a composite signal is adequately approximated by a random time function having a gaussian distribution of amplitudes. Furthermore, the total distortion will almost invariably be small enough that the distorted signal is also adequately approximated by such a signal.

Under these conditions, an expression of the spectral density of the output signal in terms of the filter transfer function and the spectral density of the input signal, i.e., a second-moment analysis, can provide useful engineering information. This technique wili be applied to the input-output phase relationship given by Eq. (7) using the methods described by Rice, ${ }^{(45)}$ even though the use of a gaussian process cannot be justified except as an adequate approximation to the time process. The convergence criterion developed in Appendix A requires that the Taylor's series expansion for the input signal $\exp [i \varphi(t)]$ converge uniformly in the infinite interval on $t$. Only bounded functions can be shown to satisfy such a requirement, and while the bound can be arbitrarily large, it can always be exceeded 
by a gaussian function. It is hoped that the plausibility of the results will stimulate efforts at analytical justification of the procedure.

Let $\varphi(t, \omega)$, wes, denote a sample function of a stationary, differentiable, zero-mean, gaussian process having an autocorrelation function $R_{\varphi}(t)$ and a spectral density $W_{\varphi}(f)$, where $R$ and $W$ are a Fourier pair

$$
R(t)=\int_{-\infty}^{\infty} d f W(f) e^{i 2 \pi f t}, W(f)=\int_{-\infty}^{\infty} d t R(t) e^{-i 2 \pi f t}
$$

The autocorrelation function is defined by

$$
R_{\varphi}(\tau)=E[\varphi(t, \omega) \varphi(t+\tau, \omega)]
$$

where the expectation operator $\mathrm{E}$ denotes an ensemble average. The output spectral density is determined formally from Eq. (7) by using Eq. (11) to form the autocorrelation function and then by applying Eq. (10). That is

$$
W_{\theta}(f)=\mathcal{F}_{E}[\theta(t, w) \theta(t+\tau, w)]
$$

where $\mathcal{F}$ denotes the Fourier transform operator and where the operations are applied tem-by-term to the pairings which result from using the expansion for $\theta$. If it is desired to express the results in terms of the input and output phase rates, the relationships

$$
W_{\dot{\theta}}(f)=(2 \pi f)^{2} W_{\theta}(f), \quad W_{\varphi}(f)=W_{\dot{\varphi}}(f) /(2 \pi f)^{2}
$$

(Ref. 45, Sec. 3.3) may be used. 
To facilitate the identification of the various components of the output spectrum, let the superscripts S, D and C, when applied to the output spectrum $W_{\theta}(f)$, denote, respectively, the signal, distortion and crosstalk. Then, as shown in detail in Appendix $C$, a spectral analysis of Eq. (7) yields

$$
\begin{aligned}
& W_{\theta}^{S}(f)=|G(f)|^{2} W_{\phi}(f) \\
& W_{\theta}^{D}(f)=2 W_{\varphi}(f) \int_{-\infty}^{\infty} d \rho W_{\varphi}(\rho) \\
& x\left\{\operatorname{Re} G(\rho) G(-\rho-f) G(f)-|G(f)|^{2}|G(\rho)|^{2}\right\} \\
& \mathrm{W}_{\theta}^{\mathrm{C}}(\mathrm{f})=\frac{1}{6} \int_{-\infty}^{\infty} \mathrm{d} \rho \int_{-\infty}^{\infty} \mathrm{d} \sigma \mathrm{W}_{\varphi}(\mathrm{f}-\rho-\sigma) \mathrm{W}_{\varphi}(\rho) \mathrm{W}_{\varphi}(\sigma) \\
& x \mid 2 G(f-\rho-\sigma) G(\rho) G(\sigma)-G(f-\rho-\sigma) G(\rho+\sigma) \\
& -G(\rho) G(f-\rho)-G(\sigma) G(f-\sigma)+\left.G(f)\right|^{2}
\end{aligned}
$$

The origins of these components in the expansion of Eq. (7) can be seen by designating the first three terms of the expansion as $\mathrm{S}, \mathrm{D}_{3}$ and $\mathrm{D}_{5}$, respectively. The leading term is recognized as the signal component and the next two as the third- and fifth-order distortion terms. In this notation, the signal component of the output, $W_{\theta}^{S}(f)$, is given by the $S \times S$ term in Eq. (12) and is seen to involve only a simple linear operation on the input spectrum $W_{\varphi}(f)$. Also, it is complete in the sense that no further contributions can be made to it by higher-order terms in the expansion. 
The distortion and crosstalk components of the output will generally involve operations of all higher orders on the input. Those given in Eq. (14) constitute first-order contributions inasmuch as they are the leading or principal terms of their type. The first-order distortion, $W_{\theta}^{D}(f)$, is given by the $S \times D_{3}$ terms. The $S \times D_{5}$ and some of the $\mathrm{D}_{3} \times \mathrm{D}_{3}$ terms give rise to second-order distortion components which are neglected; the first-order crosstalk, $W_{\theta}^{C}(f)$, then comes from the balance of the $\mathrm{D}_{3} \times \mathrm{D}_{3}$ terms.

This distinction between the distortion and crosstalk derives from the presence of the input spectrum, $W_{\varphi}(f)$, as a multiplicative factor in some of the output spectral terms. These terms, which are designated as distortion, are unique in that their contribution to a given frequency interval in the output is directly related to the amount of input in that same frequency interval; in particular, if there is no input, there will be no output. The contributions of the other components to a given frequency interval in the output are relatively insensitive to the presence or absence of an input in that same frequency interval, since they result from convolutions of the input spectrum and the filter transfer function. By analogy with the intermodulation discernible on an idle telephone channel, these contributions are referred to as crosstalk. Although the crosstalk level is frequently less than the distortion, it is often more objectionable subjectively and therefore constitutes an important system performance criterion. 
IV. NUMERICAL EXAMPLE--FM WITH UNIFORM BASEBAND THROUGH

\section{A SINGLE-POLE FILTER}

As mentioned in the introduction, one of the most important filters to which the foregoing analysis can be applied is the singlepole filter. The nomalized equivalent low-pass transfer function of this filter is given by

$$
G(f)=\frac{1}{1+i 2 \pi f}=\frac{1}{I+i \underline{f}}, \quad \underline{f}=\frac{f}{f}
$$

where $f_{c}$ is the $3-d b$ cutoff frequency (which corresponds to the $3-d b$ half-bandwidth of the original band-pass filter). Substituting Eq. (15) into Eqs. (14) yields, after some tedious but straightforward algebra

$$
\begin{aligned}
& W_{\theta}^{S}(f)=\frac{W_{\varphi}(f)}{1+\underline{f}^{2}} \\
& W_{\theta}^{D}(f)=\frac{4 \underline{f}^{2} W_{\varphi}(f)}{1+\underline{f}^{2}} \\
& \times \int_{-\infty}^{m} \frac{d \rho \underline{\rho}^{2} w_{\varphi}(\rho)}{\left(1+\underline{\rho}^{2}\right)\left[1+(\underline{\rho}+\underline{f})^{2}\right]\left[1+(\underline{\rho}-\underline{f})^{2}\right]} \\
& W_{\theta}^{C}(f)=\frac{1}{6\left(1+\underline{f}^{2}\right)} \int_{-\infty}^{\infty} d \rho \int_{-\infty}^{\infty} d \sigma W_{\varphi}(f-\rho-\sigma) W_{\varphi}(\rho) W_{\varphi}(\sigma)(\underline{f}-\rho-\sigma)^{2} \underline{\rho}^{2} \underline{\sigma}^{2} \\
& x \frac{4+4 \underline{\underline{f}}^{2}+4 \underline{f}(\underline{\rho}+\underline{\sigma})(\underline{f}-\underline{\rho})(\underline{f}-\underline{\sigma})+(\underline{\rho}+\underline{\underline{\sigma}})^{2}(\underline{\underline{f}}-\underline{\rho})^{2}\left(\underline{\underline{f}-\underline{\sigma})^{2}}\right.}{\left[1+(\underline{f}-\underline{\rho}-\underline{\sigma})^{2}\right]\left(1+\underline{\sigma}^{2}\right)\left(1+\underline{\rho}^{2}\right)\left[1+(\underline{\rho}+\underline{\sigma})^{2}\right]\left[1+(\underline{f}-\underline{\rho})^{2}\right]\left[1+(\underline{f}-\underline{\sigma})^{2}\right]}
\end{aligned}
$$

where the underlined quantities are normalized to $\mathrm{f}_{\mathrm{c}}$. 
An input signal of particular interest is one which is frequencymodulated by a gaussian signal having a uniform spectral density in $(A, B) c p s$, where $A \ll B$, since such a baseband closely approximates a frequency-division-multiplexed, multi-channel communication signal. The spectral density of the input phase rate can then be written

$$
W_{\dot{\varphi}}(f)=\left\{\begin{array}{cc}
\frac{(2 \pi D)^{2}}{2(B-A)}, & A \leq|f| \leq B \\
0, & f \text { elsewhere }
\end{array}\right.
$$

where $D$ is the rms deviation of instantaneous frequency and where, from Eq. (13), the spectral density of the input phase becomes

$$
W_{\varphi}(f)=\left\{\begin{array}{cl}
\frac{D^{2}}{2(B-A) f^{2}} & , A \leq|f| \leq B \\
0, & f \text { elsewhere }
\end{array}\right.
$$

It is convenient, for simplicity, to set the lowest baseband frequency A equal to zero despite the fact that the rms phase (i.e., the square root of the integral of $W_{\varphi}(f)$ over all f) then does not exist. The expansion for the output phase is, of course, meaningless in that case, but the corresponding expansion for the output phase rate apparently remains valid. However, the only justification given here is that if the spectral density of the output phase rate is computed from Eqs. (13) and (16) using Eq. (18) for $W_{\varphi}(f)$ and if the limiting values are taken as $A$ approaches zero, then the results have 
meaning and are identical to those obtained by simply setting A equal to zero. Consequently, the input phase rate and phase spectra will be taken as

$$
\begin{aligned}
& W_{\dot{\varphi}}(f)=\frac{2 \pi^{2} D^{2}}{B} \\
& W_{\varphi}(f)=\frac{D^{2}}{2 B f^{2}}, \quad|f| \leq B
\end{aligned}
$$

and zero elsewhere.

Substituting $W_{\varphi}(f)$ from Eq. (19) into Eqs. (16) and using Eq. (13) to obtain the spectra for the output phase rate then yields

$\frac{W_{\dot{\theta}}^{S}(f)}{D^{2} / f}=\frac{2 \pi^{2}}{\underline{B}\left(1+\underline{f}^{2}\right)}, \quad|f| \leq B$

$\frac{\mathrm{W}_{\dot{\dot{\theta}}}^{\mathrm{D}}(\mathrm{f})}{\mathrm{D}^{4} / \mathrm{f}_{\mathrm{c}}^{3}}=\frac{8 \pi^{2} \underline{\mathrm{f}}^{2}}{\underline{\mathrm{B}}^{2}\left(1+\underline{f}^{2}\right)} \int_{0}^{\frac{\mathrm{B}}{2}} \frac{\mathrm{d} \underline{\rho}}{\left(1+\underline{\rho}^{2}\right)\left[1+(\underline{\rho}+\underline{f})^{2}\right]\left[1+(\underline{\rho}-\underline{f})^{2}\right]}, \quad|f| \leq B$

$\frac{w_{\dot{\theta}}^{C}(f)}{D^{6} / f_{c}^{5}}=\frac{\pi^{2} \underline{f}^{2}}{12 \underline{B}^{3}\left(1+\underline{f}^{2}\right)} \int \mathrm{d} \rho \int \mathrm{d} \underline{\sigma}$

$$
\times \frac{4+4 \underline{f}^{2}+4 \underline{f}(\underline{\rho}+\underline{\sigma})(\underline{f}-\underline{\rho})(\underline{f}-\underline{\sigma})+(\underline{\rho}+\underline{\sigma})^{2}(\underline{f}-\underline{\rho})^{2}(\underline{f}-\underline{\sigma})^{2}}{\left[1+(\underline{f}-\underline{\rho}-\underline{\sigma})^{2}\right]\left(1+\underline{\sigma}^{2}\right)\left(1+\underline{\rho}^{2}\right)\left[1+(\underline{\rho}+\underline{\sigma})^{2}\right]\left[1+(\underline{f}-\underline{\rho})^{2}\right]\left[1+\left(\underline{\left.\underline{f}-\underline{\sigma})^{2}\right]}\right.\right.}
$$

where it is understood that the terms vanish in those frequency intervals in which they are not defined and where underlining indicates nomalization to $f_{c}$. The region of integration for the crosstalk term 
is shown in Fig. 1. Since spectral densities are even functions of frequency, these spectra will be plotted subsequently only for positive frequencies.

The spectral density of the signal component of the output phase rate given by the first of Eqs. (20) is plotted as a function of the relative baseband frequency, $f / B$, for a number of baseband-to-filter half-bandwidth ratios, $B / f_{C}$, in Fig. 2, and in decibels in Fig. 3. As might be expected, the output spectral density is fairly uniform when the filter is wide, i.e., $B / f_{c}$ small. As the filter is narrowed, the output takes the shape of the filter power response and, indeed, the spectral density is down $3 \mathrm{db}$ at the highest baseband frequency for a filter for which $B / f_{C}=1$.

The spectral density of the distortion component of the output phase rate given by the second of Eqs. (20) can be integrated by expanding the integrand in partial fractions. The result is

$$
\begin{aligned}
\frac{W_{\dot{\theta}}^{D}(f)}{D^{4} / f_{c}^{3}}= & \frac{8 \pi^{2}}{\underline{B}^{2}\left(\underline{f}^{2}+1\right)\left(\underline{f}^{2}+4\right)}\left\{\tan ^{-1} \underline{B}+\frac{3 \underline{f}}{8\left(\underline{f}^{2}+1\right)} \log \left[\frac{(\underline{B}+\underline{f})^{2}+1}{(\underline{B}-\underline{f})^{2}+1}\right]\right. \\
& \left.+\frac{\underline{f}^{2}-2}{4\left(\underline{f}^{2}+1\right)}\left[\tan ^{-1}(\underline{B}+\underline{f})+\tan ^{-1}(\underline{B}-\underline{f})\right]\right\},|f| \leq B
\end{aligned}
$$

which is plotted in Figs. 4 and 5. The distortion increases with baseband frequency in general, going as the square of the frequency for wide filters. A drop-off at the higher frequencies, resembling the skirt of the filter power response, occurs as the filter is narrowed, i.e., as $f_{c}$ is decreased thereby increasing $B / f_{c}$. 


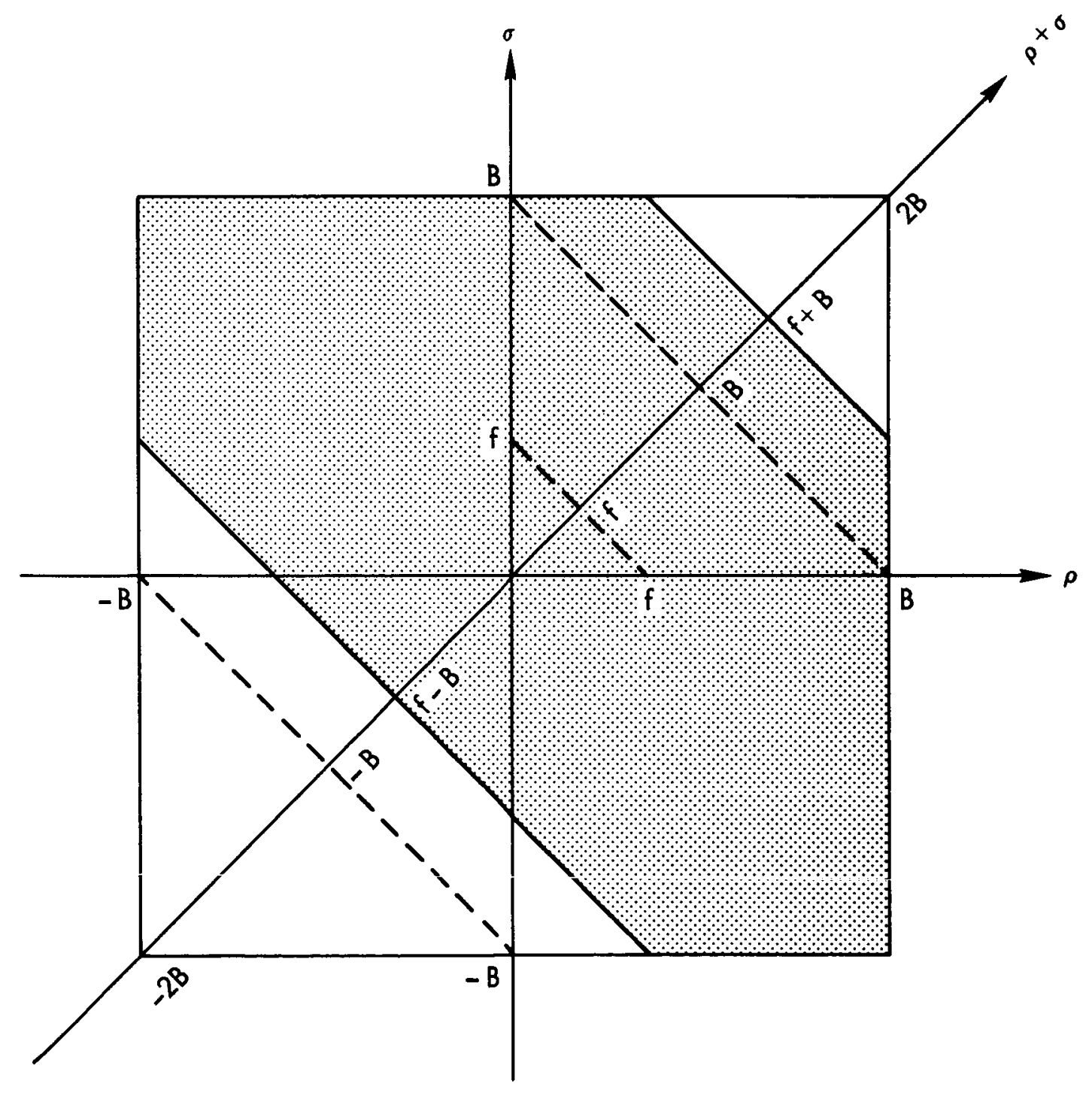

Fig. 1-Region of integration for crosstalk computation 


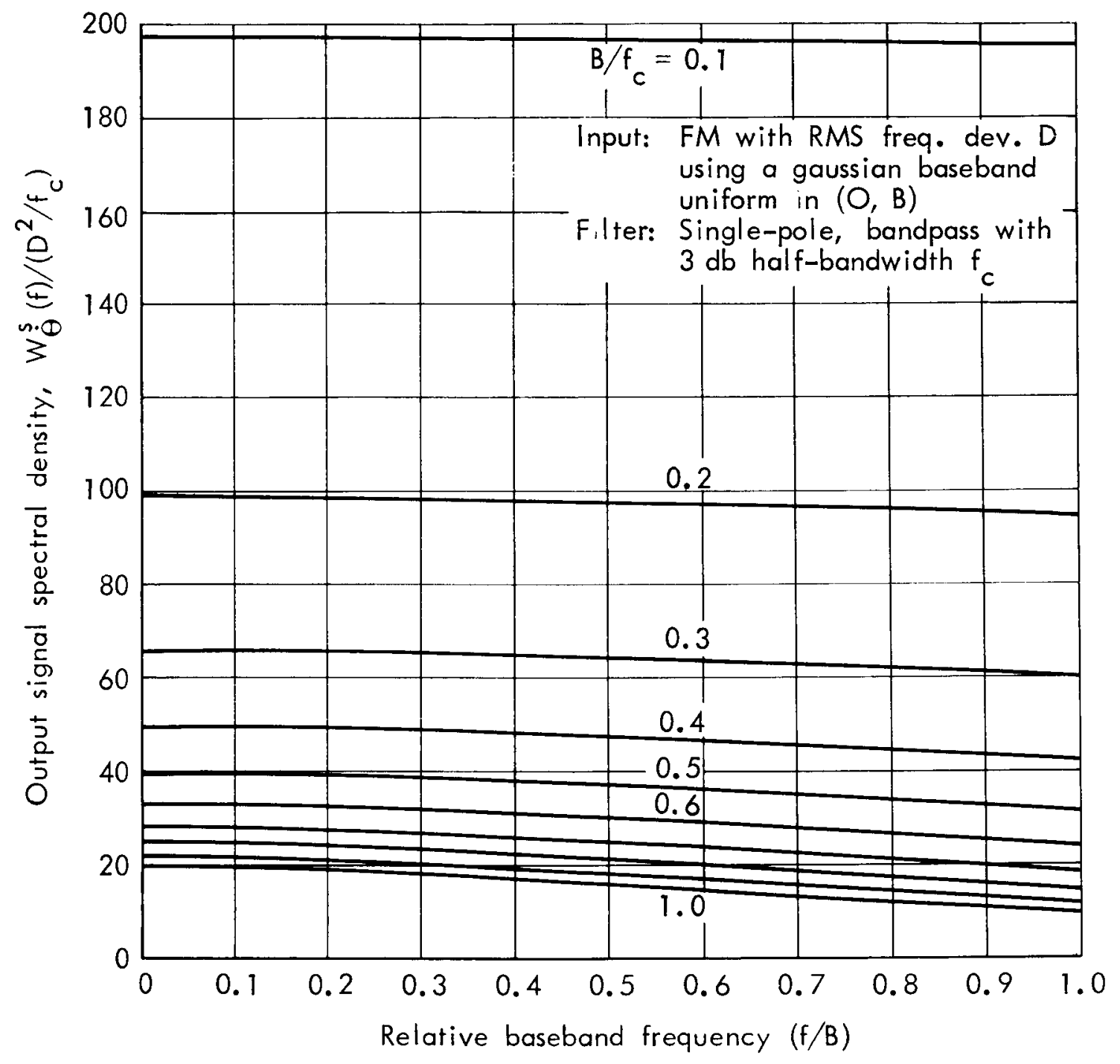

Fig.2-Output signal spectral density 


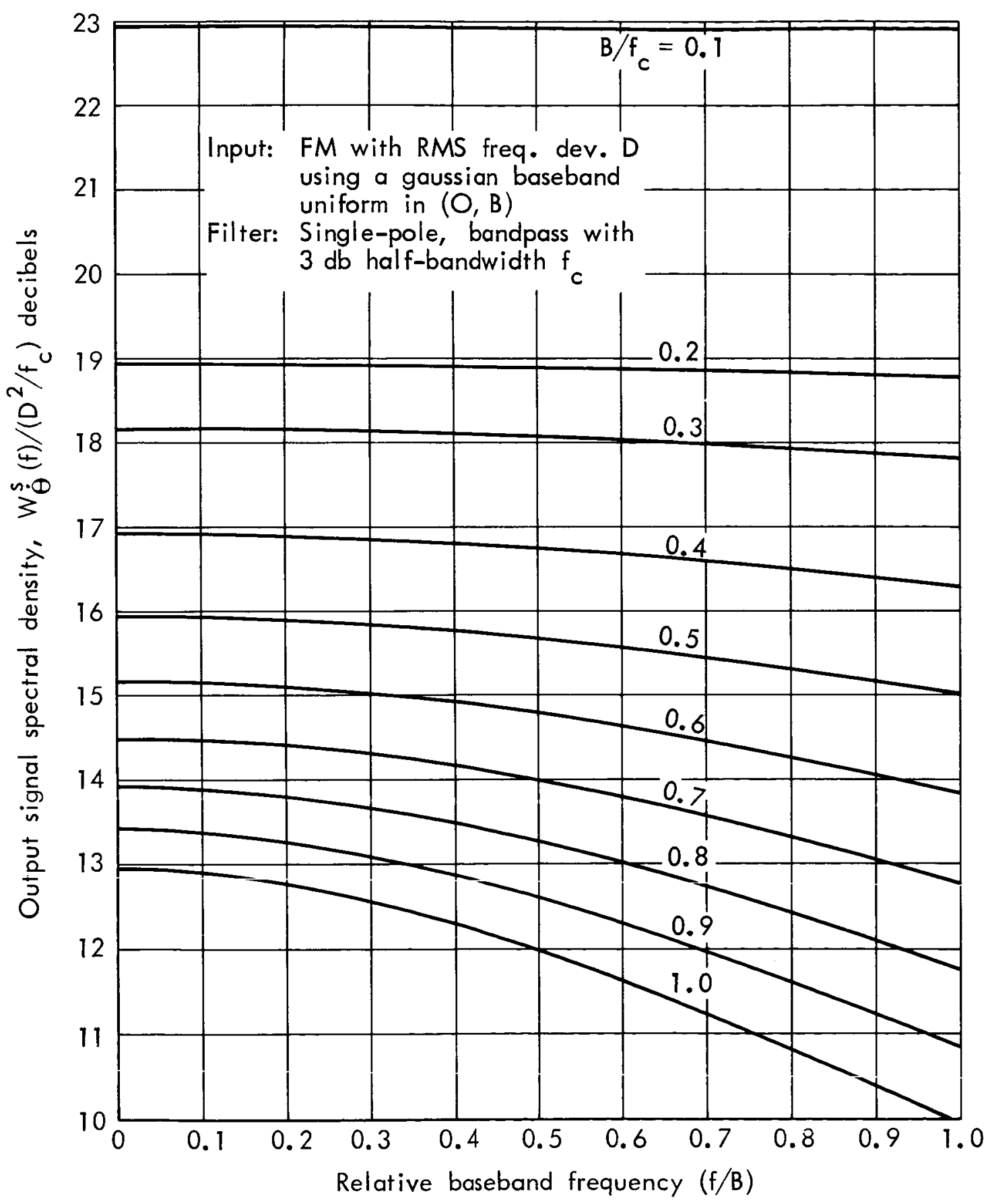

Fig.3-Output signal spectral density, decibels 


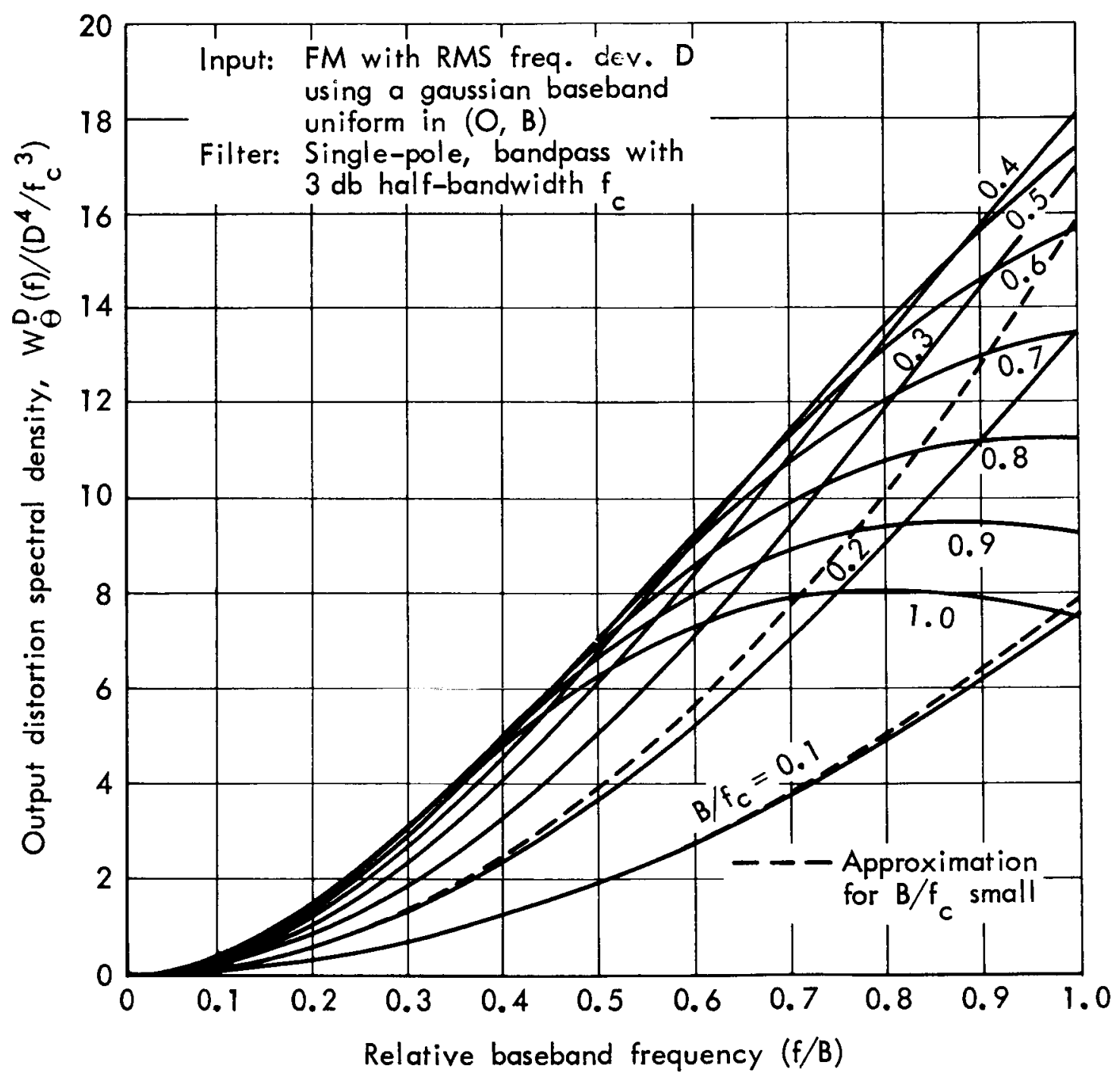

Fig.4-Output distortion spectral density 


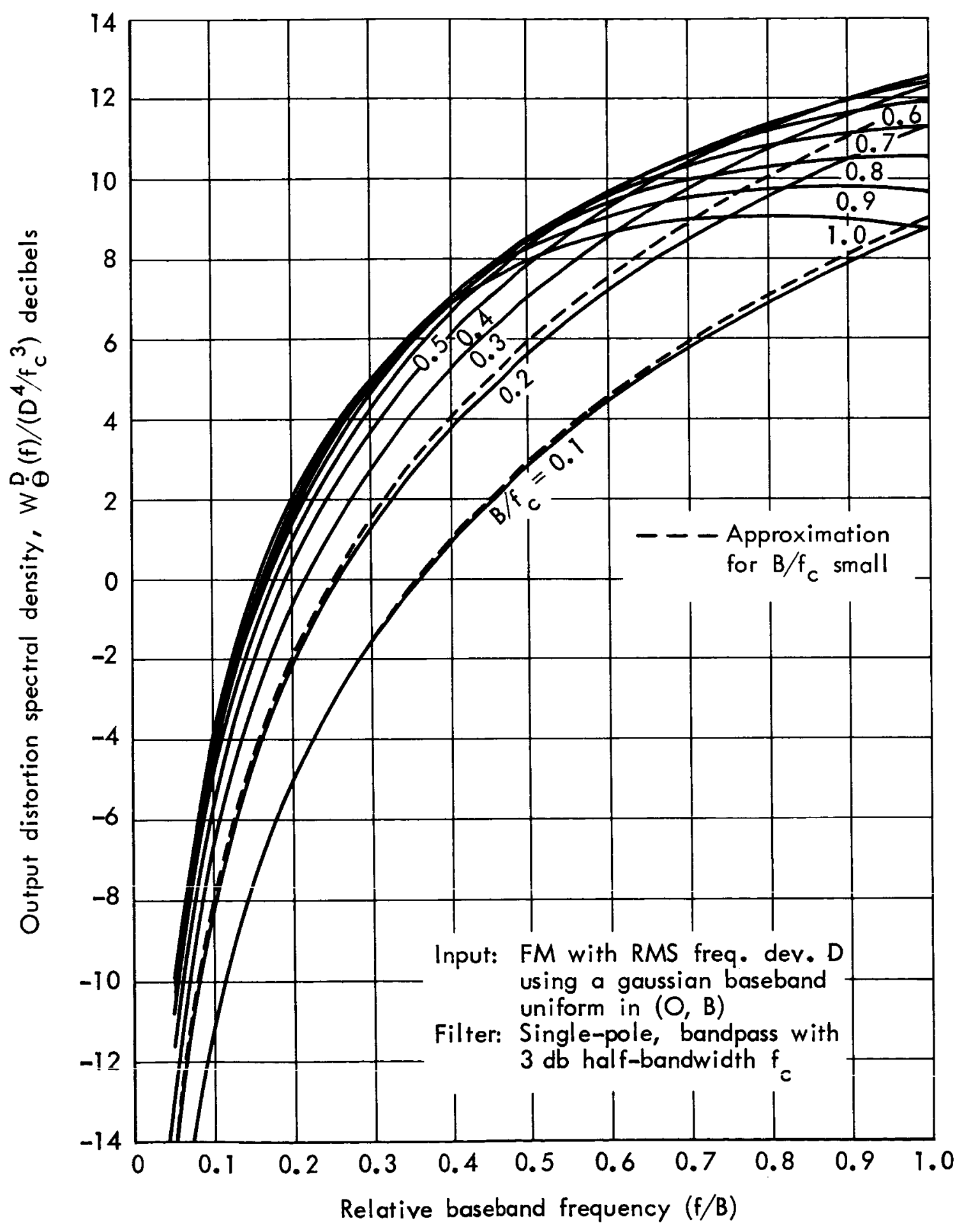

Fig.5-Output distortion spectral density, decibels 
In principle, at least one of the integrations for the crosstalk can be performed by the same procedure of expanding by partial fractions. However, the algebra required appears too formidable to warrant the effort. Even if it were accomplished, it is highly likely that the second integration would not be possible analytically. Therefore, numerical results were obtained by machine computation on the IBM 7044, using the technique of iterated integration to evaluate the double integral. ${ }^{*}$ The results are plotted in Fig. 6 , and in decibels in Fig. 7.

Like the distortion, the crosstalk tends to zero at the lower end of the baseband and, in general, increases with frequency within the baseband. Also, the effect of the filter power response is again evident for narrow filters. Typical of a third-order spectral density, the crosstalk spectrum extends to three times the highest baseband frequency and falls smoothly to zero. The signal and distortion spectra both vanish abruptly beyond the highest baseband frequencies since they both contain the input signal spectrum as a multiplicative factor.

Useful approximations to these spectra can be obtained by assuming the highest baseband frequency B sufficiently small in comparison with the filter $3-d b$ half-bandwidth, $f_{c}$, so that variations of the denominator terms in Eqs. (16) over the regions of integration can be neglected. Simple integration then yields

\footnotetext{
* The Romberg approximation to the definite integral was employed with a relative difference between successive approximations of $10^{-3}$.
} 


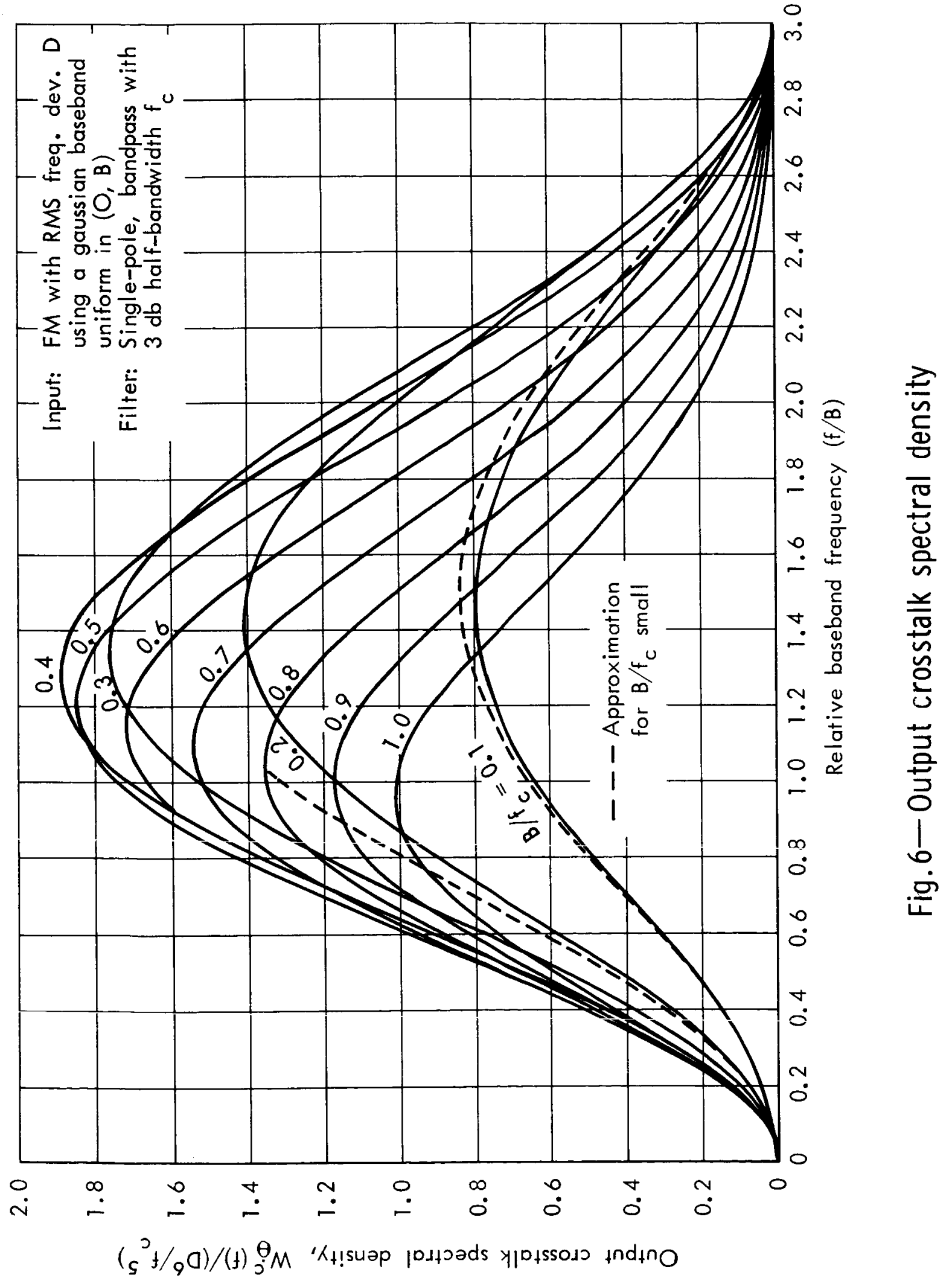




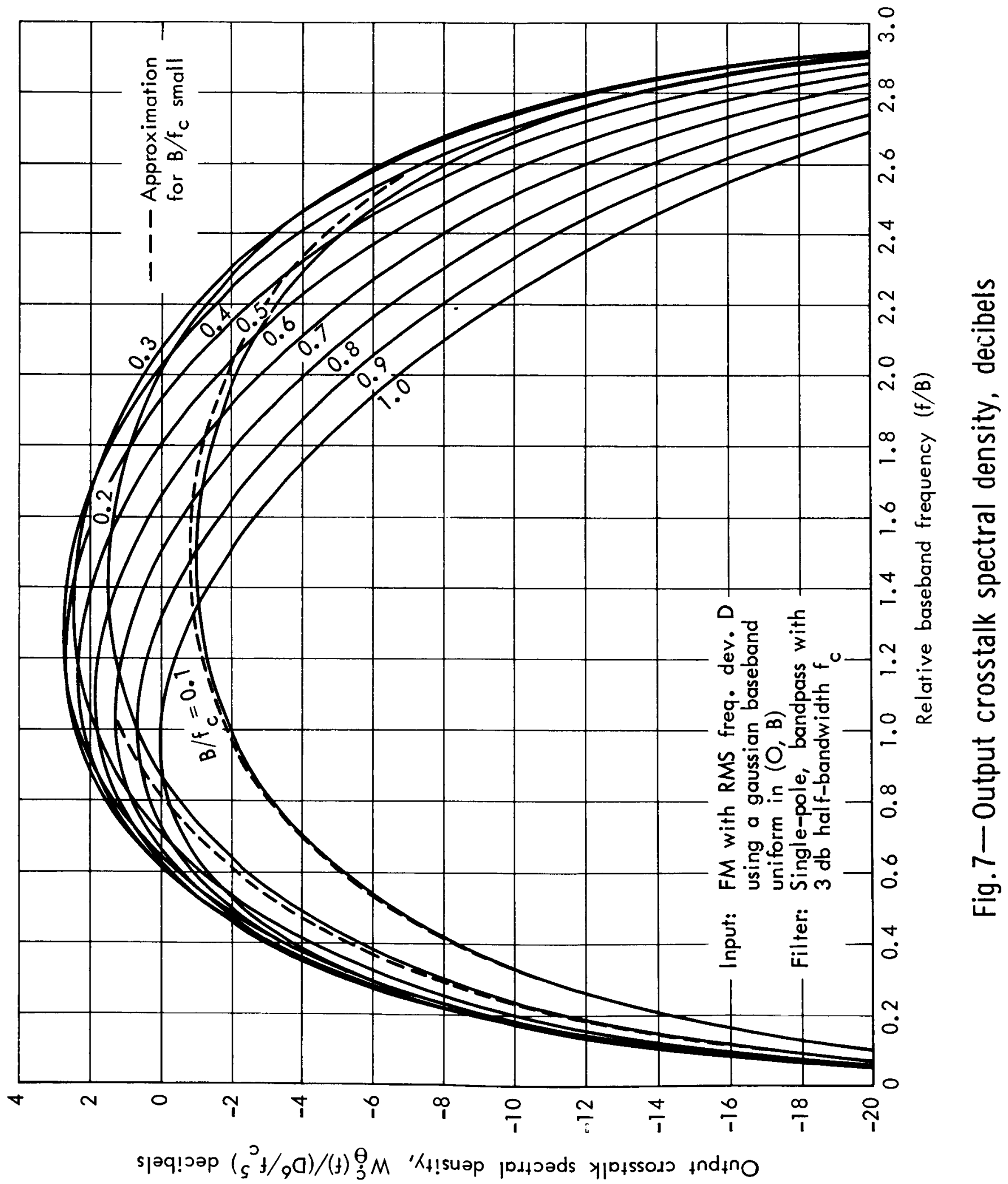




$$
\begin{aligned}
& \frac{\mathrm{W}_{\dot{\theta}}^{S}(f)}{\mathrm{p}^{2} / \mathrm{f}} \cong \frac{2 \pi^{2}}{\underline{B}}, \quad|f| \leq B \\
& \frac{W_{\dot{\theta}}^{D}(f)}{D^{4} / f_{c}^{3}} \cong \frac{8 \pi^{2} \underline{E}^{2}}{\underline{B}}, \quad|f| \leq B \\
& \frac{W_{\dot{\theta}}^{C}(f)}{D}= \begin{cases}\frac{\pi^{2} \underline{f}^{2}}{3 \underline{B}^{3}}\left(3 \underline{B}^{2}-\underline{f}^{2}\right), & |f| \leq B \\
\frac{\pi^{2} \underline{f}^{2}}{6 \underline{B}^{3}}(3 \underline{B}-|f|)^{2}, & B \leq|f| \leq 3 B\end{cases}
\end{aligned}
$$

These spectra exceed the correct values slightly but do not differ significantly for values of $B / f_{c}$ up to about 0.2. They are shown in Figs. 4 to 7 as dashed lines for $B / \mathrm{f}_{\mathrm{C}}=0.1$ and 0.2 .

The ratios of signal-to-distortion and signal-to-crosstalk spectral densities are shown in Figs. 8 and 9. These spectral ratios are quite similar in shape and decrease steadily across the baseband. The signal-to-crosstalk spectral ratio exceeds the signal-to-distortion spectral ratio by a substanlial amount for cases of practical interest, being about $10 \mathrm{db}$ greater even when the rms frequency deviation equals the filter 3-db half-bandwidth, i.e., $D / f_{c}=1$. The approximations of Eqs. (22) can also be used to obtain simple formulations of these ratios valid for $\mathrm{B} / \mathrm{f}_{\mathrm{c}}$ small. These become

$$
\begin{array}{ll}
\frac{w_{\dot{\theta}}^{S}(f)}{W_{\dot{\theta}}^{D}(f)}=\frac{1}{4 \underline{D}^{2} \underline{f}^{2}} & ,|f| \leq B \\
\frac{W_{\dot{\theta}}^{S}(f)}{W_{\dot{\theta}}^{C}(f)}=\frac{6 \underline{B}^{2}}{\underline{f}^{2} \underline{D}^{4}\left(3 \underline{B}^{2}-\underline{f}^{2}\right)}, & |f| \leq B
\end{array}
$$




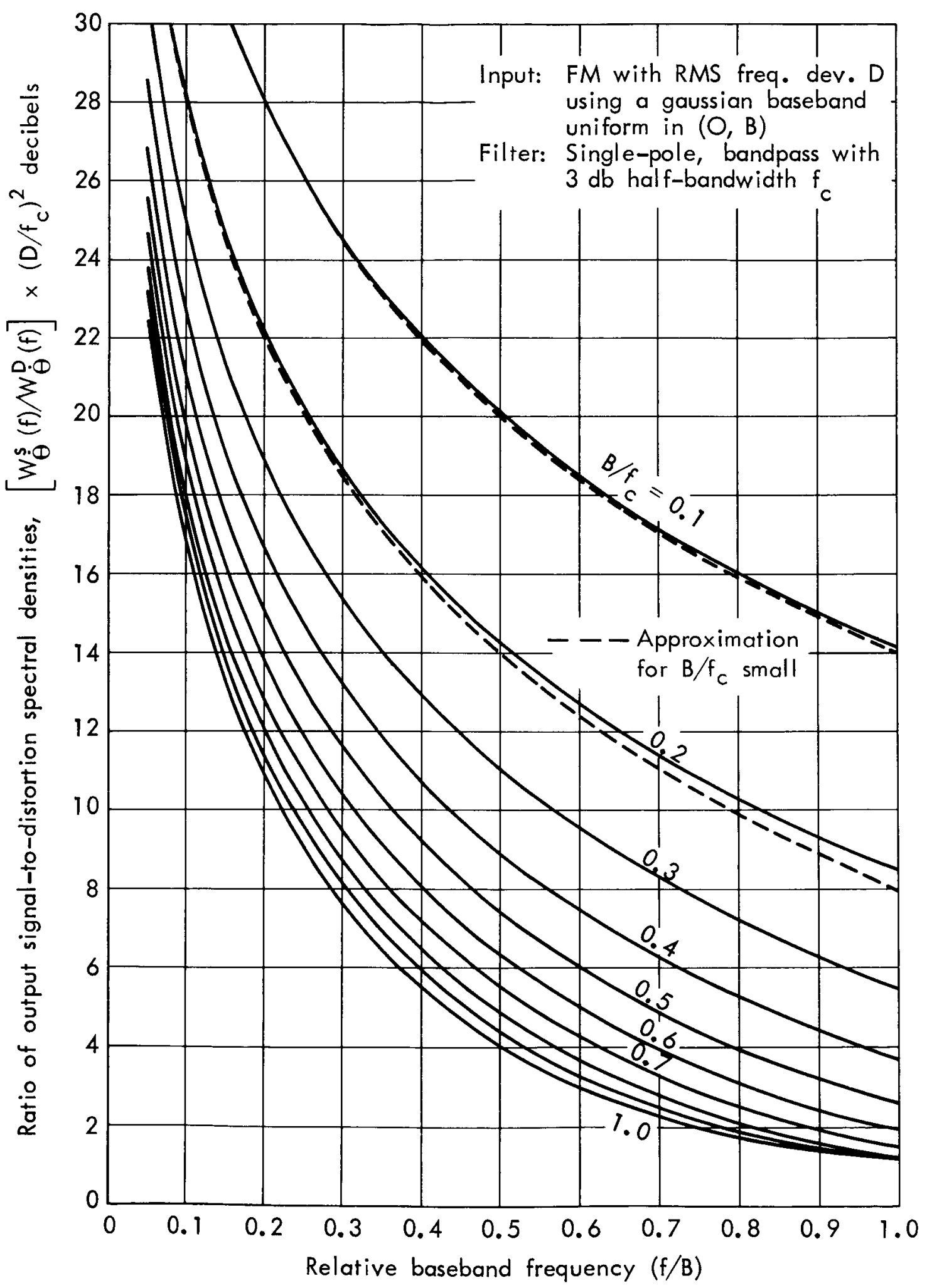

Fig.8-Ratio of output signal-to-distortion spectral densities, decibels 


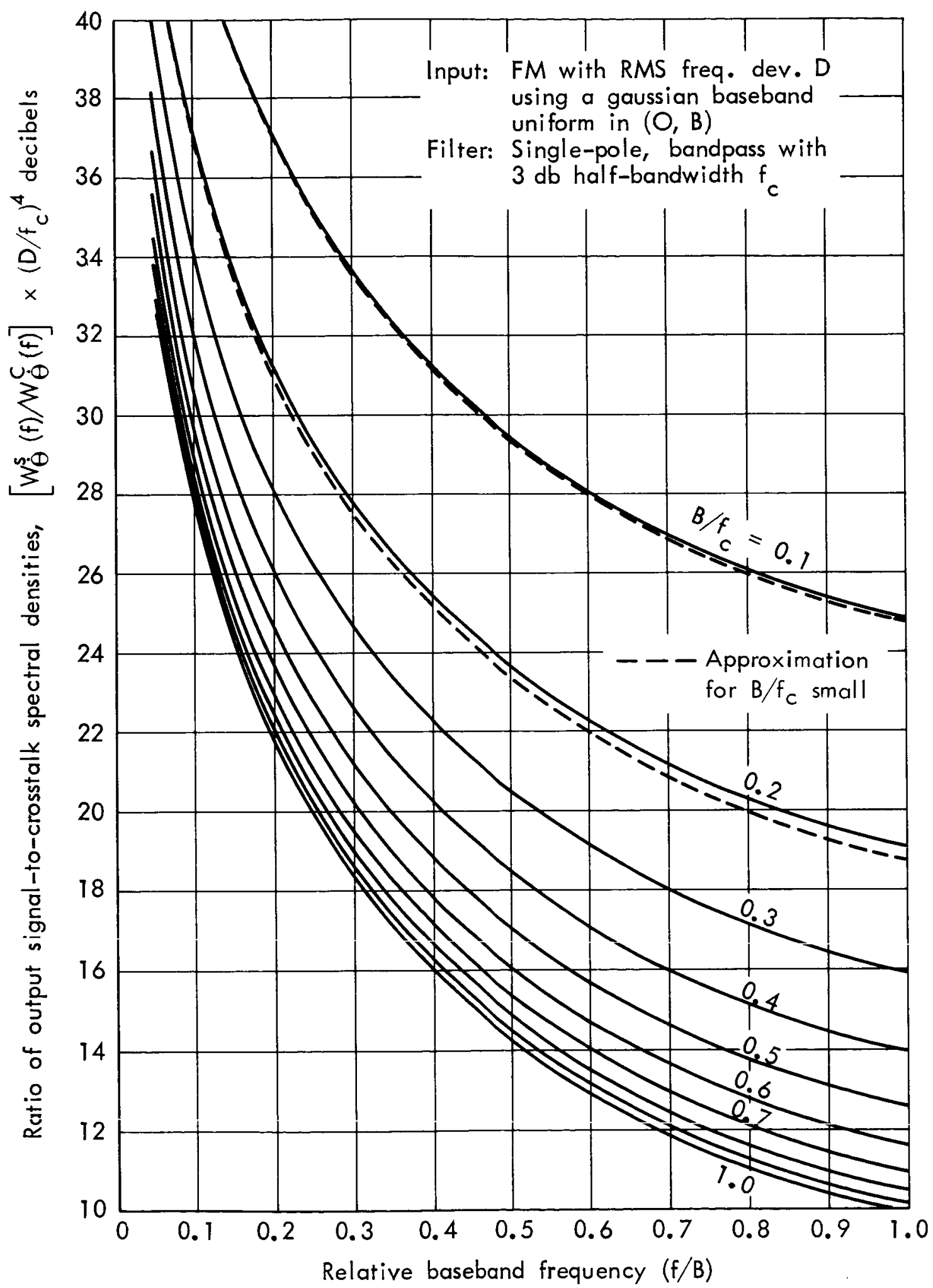

Fig.9-Ratio of output signal-to-crosstalk spectral densities, decibels 
and are shown as dashed lines on Figs. 8 and 9 for the two lower values of $B / f_{c}$.

Finally, the ratio of total signal to total distortion, SDR, is plotted against the highest normalized baseband frequency, $B / f_{c}$, in Fig. 10. The total signal is simply the integral of the first of Eqs. (20). The total distortion was computed numerically from Eq. (21) on the RAND JOSS* computer using a two-point gaussian integration with 20 intervals. The approximations of Eq. (22) may also be integrated from $-B$ to $B$ to yield

$$
\mathrm{SDR}=\frac{3}{4 \underline{D}^{2} \underline{B}^{2}}
$$

which is valid for $B / f_{C}$ small and is shown on Fig. 10 as a dashed line. It may be conjectured that if this ratio is large, then the decreasing sizes of distortion and crosstalk relative to signal will insure that the higher-order contributions of distortion and crosstalk can be safely neglected. It is on this basis that the foregoing theory is represented as an accurate measure of the distortion and crosstalk with the only condition being that the total distortion be sma 11.

For example, from Fig. 10, the signal-to-distortion ratio equals $10 \mathrm{db}$ for the filter and input spectrum being considered if the filter half-bandwidth $f_{c}$ equals 2 Mc, the rms frequency deviation $D$ equals $1.4 \mathrm{Mc}$ and the highest baseband frequency $\mathrm{B}$ equals $1 \mathrm{Mc}$, i.e., $\mathrm{D} / \mathrm{f}_{\mathrm{c}}=$ $0.7 \Rightarrow-1.5 \mathrm{db}$ and $\mathrm{B} / \mathrm{f}_{\mathrm{c}}=0.5$. Then, from Fig. 8, the signal-to-distortion spectral ratio at the upper end of the baseband (the worst channel) is $2.6+3=5.6 \mathrm{db}$ and, from Fig. 9, the corresponding signal-to-crosstalk

*JOSS is the trademark and service mark of The RAND Corporation for its on-line time-shared computer program and services using that program. 


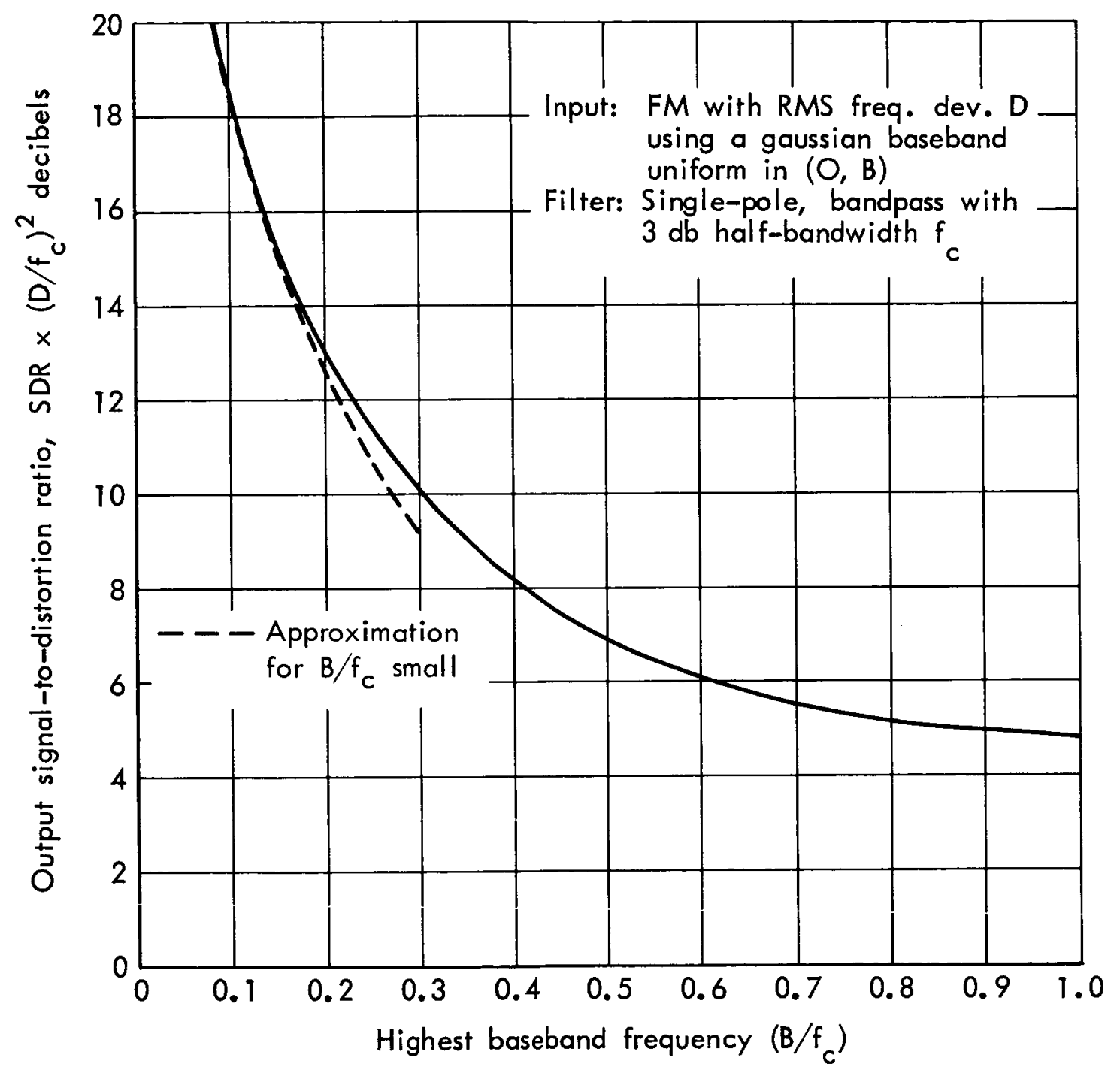

Fig. 10-Output signal-to-distortion ratio, decibels 
spectral ratio is $12.6+6=18.6 \mathrm{db}$. This is, of course, a somewhat extreme example of a virtually unusable channel but serves nevertheless to indicate the wide range of applicability of the results.

As a more practical example, consider an FM signal, modulated by a uniform baseband extending to $2.4 \mathrm{Mc}$ and having an $\mathrm{ms}$ deviation of $20 \mathrm{Mc}$ in the RF channel. Assume a frequency feedback receiver having a feedback factor of $20 \mathrm{db}$, uniform across the baseband, and a single-pole IF filter with a 3 -db half-bandwidth of 4 Mc. The ms frequency deviation in the IF channel is then $2 \mathrm{Mc}$ and the system parameters become $\mathrm{B}=2.4 \mathrm{Mc}, \mathrm{D}=2 \mathrm{Mc}$ and $\mathrm{f}_{\mathrm{C}}=4 \mathrm{Mc}$, yielding the ratios $B / f_{C}=0.6$ and $D / f_{C}=0.5 \Rightarrow-3 \mathrm{db}$. Recalling that the distortion in a linear feedback network is reduced by the feedback factor, it follows from Fig. 10 that the signal-to-distortion ratio $\operatorname{SDR}=6.1+6+20=32.1 \mathrm{db}$. The signal-to-distortion spectral ratio at the upper end of the baseband becomes $1.9+6+20=27.9 \mathrm{db}$ from Fig. 8 and the corresponding signal-to-crosstalk spectral ratio is $11.6+12+20=43.6 \mathrm{db}$ from Fig. 9 .

It is seen that the distortion and crosstalk remain substantial in tems of the criteria for high-fidelity telephony. Further improvements can be obtained only by increasing the feedback, which poses severe practical problems in such wide-band circuitry, or by widening the IF filter, which vitiates the threshold reducing advantage of the feedback process. 
Appendix A

\section{EXPANSION OF OUTPUT PHASE}

To expand the output phase given by Eq. (6) use the series

$$
\tan ^{-1} x=\frac{x}{1+x^{2}}\left[1+\frac{2}{3}\left(\frac{x^{2}}{1+x^{2}}\right)+\frac{2 \cdot 4}{3 \cdot 5}\left(\frac{x^{2}}{1+x^{2}}\right)^{2}+\ldots\right]
$$

which converges for $x^{2}<\infty$, and take $x$ as the ratio of integrals in $\mathrm{Eq} \cdot(6)$

$$
x=\frac{a}{b}=\frac{\int_{0}^{\infty} d u g(u) \sin \varphi(t-u)}{\int_{0}^{\infty} d u g(u) \cos \varphi(t-u)}
$$

Then the terms of interest in Eq. (A-1) are of the form

$\frac{x}{1+x^{2}}=\frac{a b}{a^{2}+b^{2}}=\frac{\int_{0}^{\infty} d u g(u) \sin \varphi(t-u) \int_{0}^{\infty} d u g(u) \cos \varphi(t-u)}{\left[\int_{u}^{\infty} d u g(u) \sin \varphi(t-u)\right]^{2}+\left[\int_{0}^{\infty} d u g(u) \cos \varphi(t-u)\right]^{2}}$

and

$\frac{x^{2}}{1+x^{2}}=\frac{a^{2}}{a^{2}+b^{2}}=\frac{\left[\int_{0}^{\infty} d u g(u) \sin \varphi(t-u)\right]^{2}}{\left[\int_{0}^{\infty} d u g(u) \sin \varphi(t-u)\right]^{2}+\left[\int_{0}^{\infty} d u g(u) \cos \varphi(t-u)\right]^{2}}$

The sine and cosine functions can be expanded into power series which converge for $\varphi^{2}(t)<\infty$. Let these series, or their equivalent, 


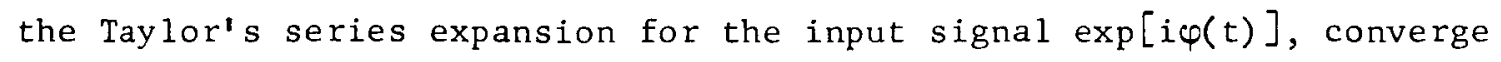
uniformly on the infinite interval on $t$. Then, since

$$
\int_{0}^{\infty} d u|g(u)|<\infty
$$

for all stable networks, the series may be integrated term by term.

The numerators in Eqs. $(\mathrm{A}-3)$ and $(\mathrm{A}-4)$ become

$$
\begin{aligned}
a b & =\left[\Phi_{1}-\frac{1}{3 !} \Phi_{3}+\frac{1}{5 !} \Phi_{5}-\ldots\right]\left[1-\frac{1}{2 !} \Phi_{2}+\frac{1}{4 !} \Phi_{4}-\ldots\right] \\
& =\Phi_{1}-\frac{1}{3 !} \Phi_{3}-\frac{1}{2 !} \Phi_{1} \Phi_{2}+\frac{1}{4 !} \Phi_{1} \Phi_{4}+\frac{1}{2 ! 3 !} \Phi_{2} \Phi_{3}+\frac{1}{5 !} \Phi_{5}+0\left(\varphi^{7}\right)
\end{aligned}
$$

and

$$
\begin{aligned}
a^{2} & =\left[\Phi_{1}-\frac{1}{3 !} \Phi_{3}+\ldots\right]^{2} \\
& =\Phi_{1}^{2}-\frac{2}{3 !} \Phi_{1} \Phi_{3}+0\left(\varphi^{6}\right)
\end{aligned}
$$

where the notation $\Phi_{n}$ is a generalization of Eq. (8)

$$
\Phi_{n}=\int_{0}^{\infty} d u g(u) \varphi^{n}(t-u)
$$

while the denominators are

$$
\begin{aligned}
a^{2}+b^{2} & =\left[\Phi_{1}-\frac{1}{3 !} \Phi_{3}+\ldots\right]^{2}+\left[1-\frac{1}{2 !} \Phi_{2}+\frac{1}{4 !} \Phi_{4}-\ldots\right]^{2} \\
& =\Phi_{1}^{2}-\frac{2}{3 !} \Phi_{1} \Phi_{3}+\ldots+1-\frac{2}{2 !} \Phi_{2}+\frac{1}{(2 !)^{2}} \Phi_{2}^{2}+\frac{2}{4 !} \Phi_{4}+\ldots \\
& =1+\Phi_{1}^{2}-\Phi_{2}-\frac{1}{3} \Phi_{1} \Phi_{3}+\frac{1}{4} \Phi_{2}^{2}+\frac{1}{12} \Phi_{4}+0\left(\varphi^{6}\right) \\
& =1+R
\end{aligned}
$$


The reciprocal of the denominators can be expressed in powers of $R$ by using the expansion

$$
(1+R)^{-1}=1-R+R^{2}-\ldots, \quad R^{2}<1
$$

which requires that the sum $a^{2}+b^{2}$ lie in the open interval $(0,2)$. It is not possible to bound the sum away from the value zero since certain functions can cause $a$ and $b$ to vanish simultaneously for some values of $t$. Thus, a necessary condition for inverting the denominator is that $a+i b, i . e .$, the output signal given by Eq. (5), not vanish. An example of a function which achieves this at $t=0$ is $\varphi(t)=$ $2 \pi k \int_{0}^{-t} g(s) d s+\lambda$, where $k$ is an integer not zero and $\lambda$ is an arbitrary constant. ${ }^{*}$ It will be assumed in the following that such functions are sufficiently singular that they can be avoided or, as will be the case when a spectral analysis is performed using an ensemble of functions, that their effect can be neglected.

To bound the denominator from above, note that it can be written

$$
\begin{aligned}
a^{2}+b^{2}= & \int_{0}^{\infty} d u \int_{0}^{\infty} d v g(u) g(v) \cos [\varphi(t-u)-\varphi(t-v)] \\
& \leq \int_{0}^{\infty} d u \int_{0}^{\infty} d v|g(u)||g(v)|=\left[\int_{0}^{\infty} \operatorname{du}|g(u)|\right]^{2}
\end{aligned}
$$

from which it follows that a sufficient condition for inverting the denominator is given by

$$
\int_{0}^{\infty} d u|g(u)|<\sqrt{2}
$$

\footnotetext{
* Private communication from 0 . A. Gross of The RAND Corporation.
} 
This condition requires that the filter impulse response be "well behaved." While the looseness of the bound used may have made the condition excessively restrictive for general use, it is satisfactory for the cases of immediate interest. For example, it is clearly satisfied by any non-negative impulse response, such as that of a firstorder filter, by virtue of the normalization of Eq. (4). Although it does not appear feasible to apply Eq. (A-12) analytically to the impulse responses of higher-order filters, it can be used graphically on plots similar to those given by Henderson and Kautz. (47) It is apparent by inspection of Fig. 9, Ref. 47, that Bessel filters of all orders satisfy Eq. (A-12). The low-order Butterworth filters, and to a lesser extent Tchebycheff filters, also appear to have sufficiently small negative-going regions in their impulse responses to satisfy the condition.

Returning to Eq. (A-9), expanding in powers of $\mathrm{R}$ according to Eq. (A-10) then yields

$$
\begin{aligned}
\left(\mathrm{a}^{2}+\mathrm{b}^{2}\right)^{-1}= & 1-\left[\Phi_{1}^{2}-\Phi_{2}-\frac{1}{3} \Phi_{1} \Phi_{3}+\frac{1}{4} \Phi_{2}^{2}+\frac{1}{12} \Phi_{4}-\ldots\right] \\
& +\left[\Phi_{1}^{4}-2 \Phi_{1}^{2} \Phi_{2}+\Phi_{2}^{2}+\ldots\right]+\ldots \\
= & 1-\Phi_{1}^{2}+\Phi_{2}+\frac{1}{3} \Phi_{1} \Phi_{3}+\frac{3}{4} \Phi_{2}^{2}-\frac{1}{12} \Phi_{4}+\Phi_{1}^{4}-2 \Phi_{1}^{2} \Phi_{2}+0\left(\varphi^{6}\right)
\end{aligned}
$$

Substituting Eqs. $(A-6)$ and $(A-13)$ into Eq. (A-3) gives the leading term in the expansion of Eq. (A-1) 


$$
\begin{aligned}
\frac{\mathrm{x}}{1+\mathrm{x}^{2}}= & (\mathrm{ab})\left(\mathrm{a}^{2}+\mathrm{b}^{2}\right)^{-1} \\
= & {\left[\Phi_{1}-\frac{1}{6} \Phi_{3}-\frac{1}{2} \Phi_{1} \Phi_{2}+\frac{1}{24} \Phi_{1} \Phi_{4}+\frac{1}{12} \Phi_{2} \Phi_{3}+\frac{1}{120} \Phi_{5}+\ldots\right] } \\
& \times\left[1-\Phi_{1}^{2}+\Phi_{2}+\frac{1}{3} \Phi_{1} \Phi_{3}+\frac{3}{4} \Phi_{2}^{2}-\frac{1}{12} \Phi_{4}+\Phi_{1}^{4}-2 \Phi_{1}^{2} \Phi_{2}+\ldots\right] \\
= & \Phi_{1}-\Phi_{1}^{3}+\Phi_{1} \Phi_{2}+\frac{1}{3} \Phi_{1}^{2} \Phi_{3}+\frac{3}{4} \Phi_{1} \Phi_{2}^{2}-\frac{1}{12} \Phi_{1} \Phi_{4}+\Phi_{1}^{5}-2 \Phi_{1} \Phi_{2}-\frac{1}{6} \Phi_{3}+\frac{1}{6} \Phi_{1}^{2} \Phi_{3}-\frac{1}{6} \Phi_{2} \Phi_{3} \\
& -\frac{1}{2} \Phi_{1} \Phi_{2}+\frac{1}{2} \Phi_{1}^{3} \Phi_{2}-\frac{1}{2} \Phi_{1} \Phi_{2}^{2}+\frac{1}{24} \Phi_{1} \Phi_{4}+\frac{1}{12} \Phi_{2} \Phi_{3}+\frac{1}{120} \Phi_{5}+\ldots \\
= & \Phi_{1}-\Phi_{1}^{3}+\frac{1}{2} \Phi_{1} \Phi_{2}-\frac{1}{6} \Phi_{3}+\frac{1}{2} \Phi_{1}^{2} \Phi_{3}+\frac{1}{4} \Phi_{1} \Phi_{2}^{2}-\frac{1}{24} \Phi_{1} \Phi_{4} \\
& +\Phi_{1}^{5}-\frac{3}{2} \Phi_{1}^{3} \Phi_{2}-\frac{1}{12} \Phi_{2} \Phi_{3}+\frac{1}{120} \Phi_{5}+0\left(\varphi^{7}\right)
\end{aligned}
$$

Similarly, substituting Eqs. (A-6), (A-7), and (A-13) into Eqs. (A-3) and (A-4) yields the second term in Eq. (A-1)

$$
\begin{aligned}
\frac{2}{3} \frac{\mathrm{x}}{1+\mathrm{x}^{2}} \frac{\mathrm{x}^{2}}{1+\mathrm{x}^{2}} & =\frac{2}{3}(\mathrm{ab}) \mathrm{a}^{2}\left(\mathrm{a}^{2}+\mathrm{b}^{2}\right)^{-2} \\
& =\frac{2}{3}\left[\Phi_{1}-\frac{1}{6} \Phi_{3}-\frac{1}{2} \Phi_{1} \Phi_{2}+\ldots\right]\left[\Phi_{1}^{2}-\frac{1}{3} \Phi_{1} \Phi_{3}+\ldots\right]\left[1-\Phi_{1}^{2}+\Phi_{2}+\ldots\right]^{2} \\
& =\frac{2}{3}\left[\Phi_{1}^{3}-\frac{1}{2} \Phi_{1}^{2} \Phi_{3}-\frac{1}{2} \Phi_{1}^{3} \Phi_{2}+\ldots\right]\left[1-2 \Phi_{1}^{2}+2 \Phi_{2}+\ldots\right] \\
& =\frac{2}{3}\left[\Phi_{1}^{3}-2 \Phi_{1}^{5}+2 \Phi_{1}^{3} \Phi_{2}-\frac{1}{2} \Phi_{1}^{2} \Phi_{3}-\frac{1}{2} \Phi_{1}^{3} \Phi_{2}+\ldots\right] \\
& =\frac{2}{3} \Phi_{1}^{3}-\frac{4}{3} \Phi_{1}^{5}+\Phi_{1}^{3} \Phi_{2}-\frac{1}{3} \Phi_{1}^{2} \Phi_{3}+0\left(\varphi^{7}\right)
\end{aligned}
$$

The third term in Eq. (A-1) is

$$
\begin{aligned}
\frac{8}{15} \frac{x}{1+x^{2}}\left(\frac{x^{2}}{1+x^{2}}\right)^{2} & =\frac{8}{15}(a b) a^{4}\left(a^{2}+b^{2}\right)^{-3} \\
& =\frac{8}{15} \Phi_{1}^{5}+0\left(\varphi^{7}\right)
\end{aligned}
$$

Summation of Eqs. $(A-14),(A-15)$, and $(A-16)$ produces, finally 


$$
\begin{aligned}
\theta(t)=\Phi_{1} & -\frac{1}{3} \Phi_{1}^{3}+\frac{1}{2} \Phi_{1} \Phi_{2}-\frac{1}{6} \Phi_{3}+\frac{1}{6} \Phi_{1}^{2} \Phi_{3}+\frac{1}{4} \Phi_{1} \Phi_{2}^{2}-\frac{1}{24} \Phi_{1} \Phi_{4} \\
& +\frac{1}{5} \Phi_{1}^{5}-\frac{1}{2} \Phi_{1}^{3} \Phi_{2}-\frac{1}{12} \Phi_{2} \Phi_{3}+\frac{1}{120} \Phi_{5}+\ldots \\
= & \Phi_{1}-\frac{1}{6}\left[\Phi_{3}-3 \Phi_{2} \Phi_{1}+3 \Phi_{1} \Phi_{1}^{2}-\Phi_{1}^{3}\right] \\
& +\frac{1}{120}\left[\Phi_{5}-5 \Phi_{4} \Phi_{1}+10 \Phi_{3} \Phi_{1}^{2}-10 \Phi_{2} \Phi_{1}^{3}+5 \Phi_{1} \Phi_{1}^{4}-\Phi_{1}^{5}\right] \\
& -\frac{1}{12}\left[\Phi_{2}-2 \Phi_{1} \Phi_{1}+\Phi_{1}^{2}\right]\left[\Phi_{3}-3 \Phi_{2} \Phi_{1}+3 \Phi_{1} \Phi_{1}^{2}-\Phi_{1}^{3}\right]+\ldots \\
=\Phi(t) & -\frac{1}{3 !} \int_{0}^{\infty} \mathrm{dug} g(u)\left[\varphi(t-u)-\Phi_{(t)}\right]^{3}+\frac{1}{5 !} \int_{0}^{\infty} \mathrm{du} g(u)\left[\varphi(t-u)-\Phi_{(t)}\right]^{5} \\
& \quad-\frac{1}{12} \int_{0}^{\infty} \mathrm{du} g(u)[\varphi(t-u)-\Phi(t)]^{2} \int_{0}^{\infty} \mathrm{du} g(u)[\varphi(t-u)-\Phi(t)]^{3}+0\left(\varphi^{7}\right)
\end{aligned}
$$

which is the desired result. The algebraic manipulations required between these steps are not obvious and, if desired, the result is most easily verified by expanding Eq. (A-17) and comparing it with the preceding lines. 


\section{Appendix B \\ TAYLOR'S SERIES EXPANSION}

The output phase function given by Eq. (6) is vector-valued, i.e., each input phase function $\varphi(t, \omega)$, wes., defines a value of the output phase function $\theta(t, \omega)$ which is a point or vector in d. Such vector-valued functions are treated by Hille and Phillips ${ }^{(44)}$ who show that under certain conditions the Taylor's series expansion of a vector-valued function $f(x)$ is given (Thms, 3.16 .2 or 3.17 .1 ) by

$$
f(x+h)=\sum_{n=0}^{\infty} \frac{1}{n !} \delta^{n}(x ; h)
$$

where (Eq. (3.16.1)) $6^{n} f(x ; h)$, the $n^{\text {th }}$ variation of $f(x)$ with increment $h$, is given by

$$
\delta^{n} f(x ; h)=\left[\frac{d^{n}}{d \zeta^{n}} f(x+\zeta h)\right]_{\zeta=0}
$$

It is also shown (Thm. 26.3.5) that $\delta^{n} f(x ; h)$ is a homogeneous polynomial of degree $\mathrm{n}$ in $\mathrm{h}$ and that, in particular (Thm, 26.3.2), $\delta \mathrm{f}(\mathrm{x} ; \mathrm{h})$ is linear in $\mathrm{h}$. Assuming differentiability, it is possible to obtain the expansion for $\theta(t)$ given by Eq. (7) by applying the duove to Eq. (6). The expansion will be evaluated at the point $\mathrm{x}=0$ and $\mathrm{h}$ will be taken as the input phase $\varphi$, i.e.

$$
\mathrm{h}_{\mathrm{u}} \equiv \varphi(\mathrm{t}-\mathrm{u})
$$


where the subscript has been introduced to identify the variable of integration. In these terms, the expansion becomes

$$
f(h)=\sum_{n=0}^{\infty} \frac{1}{n !} \delta^{n} f(0 ; h)
$$

where

$$
\delta^{n} f(0 ; h)=\left.\frac{d^{n}}{d \zeta^{n}} \tan ^{-1} \frac{\int_{0}^{\infty} d u g(u) \sin \zeta h u}{\int_{0}^{\infty} d u g(u) \cos \zeta h u}\right|_{\zeta=0}
$$

The zero-order variation is found directly from Eq. (B-5) for $\mathrm{n}=0$

$$
\delta^{0} f(0 ; h)=0
$$

The first derivative is given by

$$
\begin{aligned}
& \frac{d}{d \zeta} \tan ^{-1} \frac{a}{b} \\
& =\frac{b a^{\prime}-a b^{\prime}}{a^{2}+b^{2}} \\
& =\frac{\int_{0}^{\infty} d u g(u) \cos \zeta h_{u} \int_{0}^{\infty} d u g(u) h_{u} \cos \zeta h_{u}+\int_{0}^{\infty} d u g(u) \sin \zeta h_{\int} \int_{0}^{\infty} d u g(u) h_{u} \sin \zeta h_{u}}{\left[\int_{0}^{\infty} d u g(u) \sin \zeta h_{u}\right]^{2}+\left[\int_{0}^{\infty} d u g(u) \cos \zeta h_{u}\right]^{2}} \\
& =\frac{\int_{0}^{\infty} d u \int_{0}^{\infty} d v g(u) g(v) h u \cos \zeta\left(h_{u}-h_{v}\right)}{\int_{0}^{\infty} d u \int_{0}^{\infty} d v g(u) g(v) \cos \zeta\left(h_{u}-h_{v}\right)}
\end{aligned}
$$


so the first variation becomes

$$
\delta^{l} f(0 ; h)=\int_{0}^{\infty} d u g(u) h u
$$

where the nomalization of Eq. (4) has been used.

Further derivatives of $\mathrm{Eq} .(\mathrm{B}-7)$ are required to find the higher variations. These can be found systematically by writing Eq. (B-7) as

$$
R^{(0)}(\zeta)=\frac{N^{(0)}(\zeta)}{D^{(0)}(\zeta)}
$$

or

$$
R^{(0)}(\zeta) D^{(0)}(\zeta)=N^{(0)}(\zeta)
$$

Differentiating the product then yields higher orders of $R$ implicitly. Thus, the $\mathrm{r}^{\text {th }}$ derivative of Eq. $(\mathrm{B}-10)$ is

$$
\sum_{s=0}^{r}\left(\begin{array}{l}
r \\
s
\end{array}\right) R^{(s)}(\zeta) D^{(r-s)}(\zeta)=N^{(r)}(\zeta)
$$

which, when evaluated at zero and used recursively, yields the $n^{\text {th }}$ variation according to

$$
\delta^{n} f(0 ; h)=R^{(n-1)}(0)
$$

The expansion of Eq. (B-1I) will involve various derivacives of $\mathrm{N}$ and D. From their definition by Eqs. (B-7) and (B-9), it is seen that 


$$
N^{(m)}(0)=\left\{\begin{array}{cc}
0 & ; \text { m odd } \\
(-1)^{m / 2} \int_{0}^{\infty} d u \int_{0}^{\infty} d v g(u) g(v) h_{u}\left(h_{u}-h_{v}\right)^{m} ; m \text { even }
\end{array}\right.
$$

and

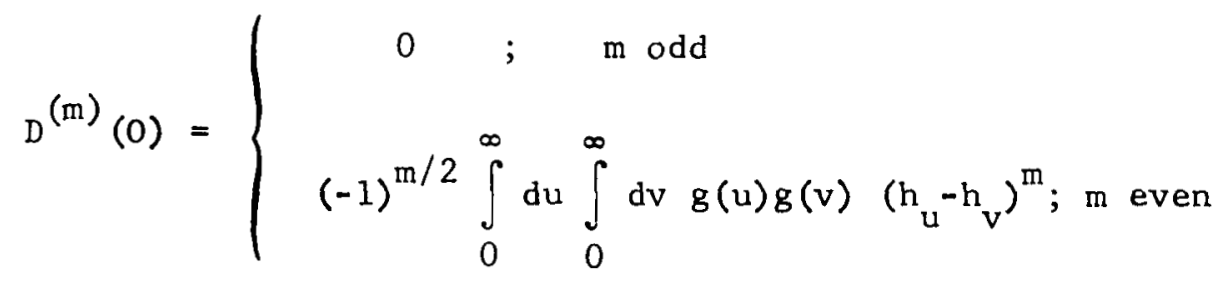

As a result, note that when $r$ is odd in Eq. (B-11) and the derivatives are evaluated at $\zeta=0$, then all the even derivatives of $R$ are paired with odd derivatives of $D$, which vanish, and the odd derivative of $\mathrm{N}$ on the right causes the sum of all products involving odd derivatives of $R$ to vanish. Commencing recursively from $R^{(1)}(0)$, which clearly vanishes, it then follows that all odd derivatives of $R$ vanish, and from Eq. (B-12), that all even variations therefore vanish, i.e.

$$
\delta^{n} f(0 ; h)=0, \quad n \text { even }
$$

In these tems, the first variation is found again by setting $r=0$ in Eq. $(B-11)$ which yields, at $\zeta=0$

$$
R^{(0)}(0)=N^{(0)}(0)=\int_{0}^{\infty} d u g(u) h_{u}
$$

wherein, from Eq. (4)

$$
D^{(0)}(0)=1
$$


Then, from Eq. (B-12)

$$
\delta^{1} f(0 ; h)=\int_{0}^{\infty} d u g(u) h u
$$

which, of course, agrees with Eq. (B-8).

To find the third variation, set $n=2$ in $E q .(B-11)$ at $\zeta=0$ and drop the vanishing odd derivatives of $R$. Then

$$
R^{(2)}(0)+R^{(0)}(0) D^{(2)}(0)=N^{(2)}(0)
$$

and

$$
\begin{aligned}
\delta^{3} f(0 ; h)=R(2)(0)= & -\int_{0}^{\infty} d u \int_{0}^{\infty} d v g(u) g(v) h_{u}\left(h_{u}-h_{v}\right)^{2} \\
& +\int_{0}^{\infty} d w g(w) h_{w} \int_{0}^{\infty} d u \int_{0}^{\infty} d v g(u) g(v)\left(h_{u}-h_{v}\right)^{2} \\
= & -\int_{0}^{\infty} d u g(u)\left[h_{u}-\int_{0}^{\infty} d v g(v) h_{v}\right]^{3}
\end{aligned}
$$

where algebraic manipulations similar to those leading to Eq. (A-17) have been performed.

The fifth variation follows from setting $n=4$ in Eq. (B-11)

$$
R^{(4)}(0)+6 R^{(2)}(0) D^{(2)}(0)+R^{(0)}(0) D^{(4)}(0)=N^{(4)}(0)
$$

so

$R^{(4)}(0)=N^{(4)}(0)-6\left[N^{(2)}(0)-N^{(0)}(0) D^{(2)}(0)\right] D^{(2)}(0)-N^{(0)}(0) D^{(4)}(0)$

Then 


$$
\begin{aligned}
& \delta^{5} f(0 ; h)=\int_{0}^{\infty} d u \int_{0}^{\infty} d v g(u) g(v) h\left(h_{u}-h_{v}\right)^{4} \\
& -6 \int_{0}^{\infty} d u \int_{0}^{\infty} d v g(u) g(v) h_{u}\left(h_{u}-h_{v}\right)^{2} \int_{0}^{\infty} d u \int_{0}^{\infty} d v g(u) g(v)\left(h_{u}-h_{v}\right)^{2} \\
& +6 \int_{0}^{\infty} d u g(u) h\left[\int_{0}^{\infty} d u \int_{0}^{\infty} d v g(u) g(v)\left(h_{u}-h_{v}\right)^{2}\right]^{2} \\
& -\int_{0}^{\infty} d u g(u) h_{u} \int_{0}^{\infty} d u \int_{0}^{\infty} d v g(u) g(v)\left(h_{u}-h_{v}\right)^{4} \\
& =\int_{0}^{\infty} d u g(u)\left[h u-\int_{0}^{\infty} d x g(x) h\right]^{5} \\
& -10 \int_{0}^{\infty} d u g(u)\left[h-\int_{0}^{\infty} d x g(x) h\right]_{x}^{2} \int_{0}^{\infty} d u g(u)\left[h-\int_{0}^{\infty} d x g(x) h x\right]^{3}
\end{aligned}
$$

where, again, a considerable number of algebraic manipulations have been performed but not shown.

The final expansion is obtained by substituting Eqs. (B-6), (B-8), $(B-15),(B-20)$ and $(B-22)$ in Eq. $(B-1)$ and using Eqs. (B-3) and $(A-8)$ to return to the original notation. Then

$$
\begin{aligned}
\theta(t)= & \Phi(t) \\
& -\frac{1}{3 !} \int_{0}^{\infty} d u g(u)[\varphi(t-u)-\Phi(t)]^{3}+\frac{1}{5 !} \int_{0}^{\infty} d u g(u)[\varphi(t-u)-\Phi(t)]^{5} \\
& -\frac{1}{12} \int_{0}^{\infty} d u g(u)[\varphi(t-u)-\Phi(t)]^{2} \int_{0}^{\infty} d u g(u)[\varphi(t-u)-\Phi(t)]^{3}+\ldots
\end{aligned}
$$

which is the desired result. 


\section{Appendix C}

\section{SPECTRAL ANALYSIS}

As suggested in Section III, let the output phase of Eq. (7) be written

$$
\theta(t)=S(t)+D_{3}(t)+D_{5}(t)+\cdots
$$

where $\mathrm{S}, \mathrm{D}_{3}$ and $\mathrm{D}_{5}$ denote the signal and third- and fifth-order components of the output, respectively. The signal component of the output phase is then obtained immediately from Eq. (12) by considering the $\mathrm{S} \times \mathrm{S}$ term and introducing the autocorrelation function of Eq. (11) and the Fourier transform of Eq. (10) as needed. Thus

$$
\begin{aligned}
& W_{\theta}^{S}(f)=\mathcal{F} E\left[\int_{0}^{\infty} d u g(u) \varphi(t-u, \omega) \int_{0}^{\infty} d v g(v) \varphi(t+\tau-v, \omega)\right] \\
& =\int_{0}^{\infty} d u \int_{0}^{\infty} d v g(u) g(v) \mathcal{F}[\varphi(t-u, w) \varphi(t+\tau-v, w)] \\
& =\int_{0}^{\infty} u \int_{0}^{\infty} d v g(u) g(v) \mathcal{H R}(\tau+u-v) \\
& =\int_{0}^{\infty} d u \int_{0}^{\infty} d v g(u) g(v) \int_{-\infty}^{\infty} d \tau R_{\varphi}(\tau+u-v) e^{-i 2 \pi f \tau} \\
& =\int_{0}^{\infty} d u \int_{0}^{\infty} d v g(u) g(v) \int_{-\infty}^{\infty} d \tau R(\tau) e^{-i 2 \pi f(\tau-u+v)} \\
& =\int_{0}^{\infty} d u g(u) e^{i 2 \pi f u} \int_{0}^{\infty} d v g(v) e^{-i 2 \pi f v} \int_{-\infty}^{\infty} d \tau R_{\varphi}(\tau) e^{-i 2 \pi f \tau} \\
& =G(-f) G(f) W_{\varphi}(f)
\end{aligned}
$$


where the Fourier transforms of Eqs. (2) and (10) are used in the last step. Finally, from the property of G given by Eq. (3)

$$
W_{\theta}^{S}(f)=|G(f)|^{2} W_{\varphi}(f)
$$

which is the first of Eqs. (14). It has been derived in some detail to illustrate the sequences of steps which are typical of those to follow.

There are two $\mathrm{SxD}_{3}$ terms in Eq. (C-1). From Eq. (12) the distortion component of the output phase then becomes

$$
\begin{aligned}
W_{\theta}^{D}(f)= & \frac{1}{3 !} F E\left\{\int_{0}^{\infty} d u g(u) \varphi(t-u, w) \int_{0}^{\infty} d v g(v)[\Phi(t+\tau, \omega)-\varphi(t+\tau-v, w)]^{3}\right. \\
& \left.+\int_{0}^{\infty} d u g(u) \varphi(t+\tau-u, w) \int_{0}^{\infty} d v g(v)[\Phi(t, w)-\varphi(t-v, w)]^{3}\right\} \\
=\frac{1}{3 !} & \int_{0}^{\infty} d u \int_{0}^{\infty} d v g(u) g(v) \mathcal{F}\left\{\varphi(t-\tau-u, w)[\Phi(t, w)-\varphi(t-v, w)]^{3}\right. \\
& \left.+\varphi(t+\tau-u, w)[\Phi(t, w)-\varphi(t-v, w)]^{3}\right\}
\end{aligned}
$$

Thus, it is necessary to consider expectations of the form $E\left\{\varphi(t \pm T-u, w)[\Phi(t, w)-\varphi(t-v, w)]^{3}\right\}$.

Now, if $F(t)$ is a zero-mean gaussian variate, then ${ }^{(48)}$ (Eq. (42b))

$$
E\left[F\left(t_{1}\right) F\left(t_{2}\right) \ldots F\left(t_{2 n}\right)\right]=\sum_{\substack{\text { ail } \\ \text { pairs }}} E\left[F\left(t_{i}\right) F\left(t_{j}\right)\right] E\left[F\left(t_{k}\right) F\left(t_{l}\right)\right] \ldots
$$


where the sum is taken over all the $1 \cdot 3 \cdot 5 \cdots(2 n-1)$ different ways the $2 n$ time points $t_{1}, t_{2}, \ldots t_{2 n}$ can be divided into $n$ pairs. Then, since $\varphi$ and $\Phi-\varphi$ are such quantities, it follows that

$$
\begin{aligned}
& E\left\{\varphi(t \pm T-u, w)[\Phi(t, w)-\varphi(t-v, w)]^{3}\right\} \\
& \quad=3 E\left\{\varphi(t \pm \tau-u, w)[\Phi(t, w)-\varphi(t-v, w]\} E\left\{[\Phi(t, w)-\varphi(t-v, w)]^{2}\right\}\right.
\end{aligned}
$$

Using Eq. (8) to eliminate $\Phi$, the first expected value in Eq. (C-6) becomes

$$
\begin{aligned}
E\{\varphi(t \pm T-u, \omega) & {[\Phi(t, \omega)-\varphi(t-v, \omega)]\} } \\
& =E\left\{\varphi(t \pm \tau-u, \omega)\left[\int_{0}^{\infty} d x g(x) \varphi(t-x, \omega)-\varphi(t-v, \omega)\right]\right\} \\
& =\int_{0}^{\infty} d x g(x) E\{\varphi(t \pm \tau-u, \omega)[\varphi(t-x, \omega)-\varphi(t-v, \omega)]\} \\
& =\int_{0}^{\infty} d x g(x) R_{\varphi}( \pm T+x-u)-R_{\varphi}( \pm T+v-u)
\end{aligned}
$$

where Eq. (11) is used to introduce the autocorrelation function $\mathrm{R}_{\varphi}$. Similarly, the second expected value in Eq. (C-6) becomes 


$$
\begin{aligned}
& E\left\{[\Phi(t, \omega)-\varphi(t-v, \omega)]^{2}\right\} \\
& \quad=E\left\{\left[\int_{0}^{\infty} d x g(x) \varphi(t-x, \omega)-\varphi(t-v, \omega)\right]\left[\int_{0}^{\infty} d y g(y) \varphi(t-y, \omega)-\varphi(t-v, \omega)\right]\right\} \\
& \quad=\int_{0}^{\infty} d x \int_{0}^{\infty} d y g(x) g(y) E[\omega(t-x, \omega) \varphi(t-y, \omega)] \\
& \quad=\int_{0}^{\infty} d x \int_{0}^{\infty} d y g(x) g(y) R(x-y)-2 \int_{0}^{\infty} d x g(x) R_{\varphi}(v-x)+R_{\varphi}(0)
\end{aligned}
$$

From Eq. (10), the third term above is

$$
\mathrm{R}_{\varphi}(0)=\int_{-\infty}^{\infty} \mathrm{d} \rho \mathrm{W}_{\varphi}(\rho)
$$

and the second term becomes

$$
\begin{aligned}
\int_{0}^{\infty} d x g(x) R_{\varphi}(v-x) & =\int_{0}^{\infty} d x g(x) \int_{-\infty}^{\infty} d \rho W_{\varphi}(\rho) e^{i 2 \pi \rho(v-x)} \\
& =\int_{-\infty}^{\infty} d \rho W_{\varphi}(\rho) G(\rho) e^{i 2 \pi \rho v}
\end{aligned}
$$

The leading term in Eq. (C-8) amounts to another integration on Eq. $(\mathrm{C}-10)$, or

$$
\begin{aligned}
\int_{0}^{\infty} d x \int_{0}^{\infty} d y g(x) g(y) R_{\varphi}(x-y) & =\int_{0}^{\infty} d x g(x) \int_{-\infty}^{\infty} d \rho W_{\varphi}(\rho) G(\rho) e^{i 2 \pi \rho x} \\
& =\int_{-\infty}^{\infty} d \rho W_{\varphi}(\rho) G(\rho) G(-\rho)=\int_{-\infty}^{\infty} d \rho W_{\varphi}(\rho)|G(\rho)|^{2}
\end{aligned}
$$


Substituting Eqs. (C-9), (C-10), and (C-11) in Eq. (C-8) then yields

$E\left\{[\Phi(t, \omega)-\varphi(t-v, \omega)]^{2}\right\}=\int_{-\infty}^{\infty} d \rho w_{\varphi}(\rho)\left[|G(\rho)|^{2}-2 G(\rho) e^{i 2 \pi \rho v}+1\right]$

and further substituting Eqs. (C-7) and (C-12) in (C-6) yields

$$
\begin{aligned}
E\{\varphi(t \pm \tau-u, \omega) & {\left.[\Phi(t, \omega)-\varphi(t-v, \omega)]^{3}\right\} } \\
=3 & \left\{\int_{0}^{\infty} \mathrm{dx} g(x) R_{\varphi}( \pm \tau+x-u)-R_{\varphi}( \pm \tau+v-u)\right\} \\
& \times\left\{\int_{-\infty}^{\infty} d \rho W_{\varphi}(\rho)\left[|G(\rho)|^{2}-2 G(\rho) e^{i 2 \pi \rho v}+1\right]\right\}
\end{aligned}
$$

When the Fourier transform operation of Eq. (C-4) is performed on Eq. (C-13), it is clear that only the first factor is involved. Its two forms are

$$
\begin{aligned}
& \mathcal{F}\left\{\int_{0}^{\infty} \mathrm{dx} g(x) R_{\varphi}(T+x-u)-R_{\varphi}(T+v-u)\right\} \\
& =\int_{0}^{\infty} d x g(x) \int_{-\infty}^{\infty} d \tau e^{-i 2 \pi f \tau}\left[R_{\varphi}(T+x-u)-R_{\varphi}(T+v-u)\right] \\
& =\left[\int_{0}^{\infty} d x g(x) e^{i 2 \pi f x}-e^{i 2 \pi f v}\right] e^{-i 2 \pi f u} \int_{-\infty}^{\infty} d \tau e^{-i 2 \pi f \tau} R_{\varphi}(\tau) \\
& =\left[G(-f)-e^{i 2 \pi f v}\right] e^{-i 2 \pi f u} W_{\varphi}(f)
\end{aligned}
$$

and, noting that $R_{\varphi}$ is an even function 


$$
\begin{aligned}
\mathcal{F}\left\{\int_{0}^{\infty} \mathrm{dx} g(\mathrm{x}) \mathrm{R}_{\varphi}(-\tau+\mathrm{x}-\mathrm{u})-\mathrm{R}_{\varphi}(-\tau+v-u)\right\} \\
=\left[G(f)-\mathrm{e}^{-i 2 \pi f v}\right] \mathrm{e}^{i 2 \pi f u} W_{\varphi}(f)
\end{aligned}
$$

Substituting Eqs. (C-13), (C-14), and (C-15) in (C-4) then yields

$$
\begin{aligned}
W_{\theta}^{D}(f)=\frac{1}{2} \int_{0}^{\infty} d u \int_{0}^{\infty} d v g(u) g(v) W_{\varphi}(f) \\
\times\left\{\left[G(-f)-e^{i 2 \pi f v}\right] e^{-i 2 \pi f u}+\left[G(f)-e^{-i 2 \pi f v}\right] e^{i 2 \pi f u}\right\} \\
\times\left\{\int_{-\infty}^{\infty} d \rho W_{\varphi}(\rho)\left[|G(\rho)|^{2}-2 G(\rho) e^{i 2 \pi \rho v}+1\right]\right\}
\end{aligned}
$$

The integrations on $u$ follow trivially.

Consider next the product of the second line of Eq. (C-16) with those functions of $\rho$ in the third line which do not contain $v, v i z$. , $|G(\rho)|^{2}+1$. The bracketed quantities, $\left[G( \pm f)-\exp \left(\mp_{i} 2 \pi f v\right)\right]$, then clearly vanish when integrated on $v$. Thus, the only term remaining is 


$$
\begin{aligned}
& W_{\theta}^{D}(f)=-W_{\varphi}(f) \int_{-\infty}^{\infty} d \rho W_{\varphi}(\rho) G(\rho) \int_{0}^{\infty} d v g(v) e^{i 2 \pi \rho v} \\
& x\left\{\left[G(-f)-e^{i 2 \pi f v}\right] G(f)+\left[G(f)-e^{-i 2 \pi f v}\right] G(-f)\right\} \\
& =-W_{\varphi}(f) \int_{-\infty}^{\infty} d p W_{\varphi}(p) G(p) \\
& x\{[G(-\rho) G(-f)-G(-\rho-f)] G(f)+[G(-\rho) G(f)-G(-\rho+f)] G(-f)\} \\
& =-W_{\varphi}(f) \int_{-\infty}^{\infty} d \rho W_{\varphi}(p) G(\rho) \\
& x\left[2|G(f)|^{2} G(-\rho)-G(-\rho-f) G(f)-G(-\rho+f) G(-f)\right]
\end{aligned}
$$

The spectrum $W$ is an even function so a change in sign on the third term permits the product of the $G$ functions to be written with the negative of the arguments of the similar product in the center term. Then, by the symmetry property of G given by Eq. (3), the sum of the last two terms in Eq. (C-17) is seen to equal twice the real part of either one. Thus, Eq. (C-17) becomes

$$
W_{\theta}^{D}(f)=2 W_{\varphi}(f) \int_{-\infty}^{\infty} d \rho W_{\varphi}(\rho)\left\{\operatorname{Re} G(\rho) G(-\rho-f) G(f)-|G(f)|^{2}|G(\rho)|^{2}\right\}
$$

which is the second of Eqs. (14). 
The terms of next higher order in Eq. (12) arise from the $S \times D_{5}$ and the $\mathrm{D}_{3} \times \mathrm{D}_{3}$ terms of $\mathrm{Eq}$. (C-1). The first of these can be recognized immediately as a second-order contribution to the distortion given by Eq. (C-18). This follows from the fact that when the expected value of the resulting sixth-order product of gaussian variates is factored into the various groups of three pairs according to Eq. (C-5), then only one of the pairs will be a function of the covariance variable $T$. When the Fourier transform of the product of the three expected values is taken, only that term will be involved and the result will yield a multiplicative factor $W_{\varphi}(f)$. In this respect, it is completely analogous to the steps leading to Eq. (C-14).

The $D_{3} \times D_{3}$ term also yields a second-order distortion contribution as well as the desired first-order crosstalk. To show this, note from Eq. (C-1) that

$$
\begin{aligned}
& D_{3} \times D_{3}=\frac{1}{(3 !)^{2}} F E\left\{\int_{0}^{\infty} d u g(u)[\varphi(t-u, \omega)-\Phi(t, \omega)]^{3}\right. \\
&\left.\times \int_{0}^{\infty} d v g(v)[\varphi(t+\tau-v, \omega)-\Phi(t+\tau, \omega)]^{3}\right\} \\
&=\frac{1}{36} 7 \int_{0}^{\infty} d u \int_{0}^{\infty} d v g(u) g(v) E\left\{[\varphi(t-u, \omega)-\Phi(t, \omega)]^{3}\right. \\
&\left.\quad x[\varphi(t+\tau-v, \omega)-\Phi(t+\tau, \omega)]^{3}\right\}
\end{aligned}
$$

For brevity, let $a$ and $b$ denote, respectively, the first and second bracketed terms in Eq. (C-19). Then, according to Eq. (C-5), the 15 different pairings of $\mathrm{E}\left(\mathrm{a}^{3} \mathrm{~b}^{3}\right)$ yield the two unique terms 


$$
E\left(a^{3} b^{3}\right)=9 E\left(a^{2}\right) E\left(b^{2}\right) E(a b)+6 E^{3}(a b)
$$

of these, only $\mathrm{E}(\mathrm{ab})$ is a function of the covariance variable $\tau$, so the first term in Eq. (C-20) contributes a second-order term to the dis-

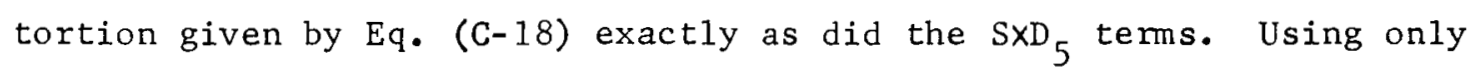
the second term of Eq. (C-20) in Eq. (C-19) then yields

$$
\begin{gathered}
W_{\theta}^{C}(f)=\frac{1}{6} 7 \int_{0}^{\infty} d u \int_{0}^{\infty} d v g(u) g(v) E^{3}\{\{\varphi(t-u, \omega)-\Phi(t, \omega)] \\
\times[\varphi(t+\tau-v, \omega)-\Phi(t+\tau, \omega)]\}
\end{gathered}
$$

and it remains to perform the indicated operations.

Noting Eq. (8) for $\Phi$, the expected value in Eq. (C-21) is given by

$$
\begin{aligned}
E(a b)= & E\left\{\left[\varphi(t-u, \omega)-\int_{0}^{\infty} d x g(x) \varphi(t-x, \omega)\right]\left[\varphi(t+\tau-v, \omega)-\int_{0}^{\infty} d x g(x) \varphi(t+\tau-x, \omega)\right]\right\} \\
= & E[\varphi(t-u, \omega) \varphi(t+T-v, \omega)]-\int_{0}^{\infty} d x g(x)\{E[\varphi(t+\tau-v, \omega) \varphi(t-x, \omega)] \\
& +E[\varphi(t-u, \omega) \varphi(t+\tau-x, \omega)]\}+\int_{0}^{\infty} d x \int_{0}^{\infty} d y g(x) g(y) E[\varphi(t-x, \omega) \varphi(t+\tau-y, \omega)] \\
= & R_{\varphi}(T+u-v)-\int_{0}^{\infty} d x g(x)\left[R_{\varphi}(T+x-v)+R(T+u-x]+\int_{0 .}^{\infty} d x \int_{0}^{\infty} d y g(x) g(y) R_{\varphi}(T+x-y)\right.
\end{aligned}
$$

where the definition of $\mathrm{R}$ given by $\mathrm{Eq} .(11)$ is used in the last step. From Eq. (10), the first term in Eq. (C-22) is simply

$$
\mathrm{T}_{1}=\int_{-\infty}^{\infty} \mathrm{d} \rho \mathrm{W}_{\varphi}(\rho) \mathrm{e}^{i 2 \pi \rho(\tau+\mathrm{u}-\mathrm{v})}
$$


and the second term, noting Eq. (2) for G, is

$$
\begin{aligned}
T_{2} & =\int_{0}^{\infty} d x g(x) \int_{-\infty}^{\infty} d \rho W_{\varphi}(\rho)\left[e^{i 2 \pi \rho(T+x-v)}+e^{i 2 \pi \rho(T+u-x)}\right] \\
& =\int_{-\infty}^{\infty} d \rho W_{\varphi}(\rho) e^{i 2 \pi \rho \tau}\left[e^{-i 2 \pi \rho v} G(-\rho)+e^{i 2 \pi \rho u} G(\rho)\right]
\end{aligned}
$$

The third term follows similarly as

$$
\begin{aligned}
T_{3} & =\int_{0}^{\infty} d x \int_{0}^{\infty} d y g(x) g(y) \int_{-\infty}^{\infty} d \rho W_{\varphi}(\rho) e^{i 2 \pi \rho(T+x-y)} \\
& =\int_{-\infty}^{\infty} d \rho W_{\varphi}(\rho) e^{i 2 \pi \rho \tau} G(\rho) G(-\rho)
\end{aligned}
$$

Substituting these into Eq. (C-22) then yields

$$
E(a b)=\int_{-\infty}^{\infty} d \rho W_{\varphi}(\rho) e^{i 2 \pi \rho T}\left[G(\rho)-e^{-i 2 \pi \rho v}\right]\left[G(-\rho)-e^{i 2 \pi \rho u}\right]
$$

Finally, substituting back into Eq. $(\mathrm{C}-21)$ in the cube gives 


$$
\begin{aligned}
& \mathrm{W}_{\theta}^{\mathrm{C}}(\mathrm{f})=\frac{1}{6} \quad \int_{0}^{\infty} \mathrm{d} u \int_{0}^{\infty} \mathrm{d} \mathrm{g} g(\mathrm{u}) \mathrm{g}(\mathrm{v}) \\
& \left.\left.x\left\{\int_{-\infty}^{\infty} \mathrm{d} \nu \mathrm{w}_{\varphi}(\nu) \mathrm{e}^{i 2 \pi \nu \tau}\left[G(\nu)-\mathrm{e}^{-i 2 \pi \nu v}\right]\left[G(-\nu)-e^{i 2 \pi \nu u}\right]\right\} \rho\right\} \sigma\right\} \\
& =\frac{1}{6} \mathcal{F} \int_{-\infty}^{\infty} \mathrm{d} \nu \int_{-\infty}^{\infty} \mathrm{d} \rho \int_{-\infty}^{\infty} \mathrm{d} \sigma \mathrm{W}_{\varphi}(\nu) \mathrm{W}_{\varphi}(\rho) \mathrm{W}_{\varphi}(\sigma) \mathrm{e}^{i 2 \pi(\nu+\rho+\sigma) \tau} \\
& x\left\{\int_{0}^{\infty} \mathrm{du} g(u)\left[G(-\nu)-e^{i 2 \pi \nu u}\right]\left[G(-\rho)-e^{i 2 \pi \rho u}\right]\left[G(-\sigma)-e^{i 2 \pi \sigma u}\right]\right\} \\
& x\left\{\int_{0}^{\infty} d v g(v)\left[G(\nu)-e^{-i 2 \pi \nu v^{-}}\right]\left[G(\rho)-e^{-i 2 \pi \rho v}\right]\left[G(\sigma)-e^{-i 2 \pi \sigma v}\right]\right\}
\end{aligned}
$$

The integrals on $\mathrm{u}$ and $\mathrm{v}$ are readily determined by expanding the integrands and applying Eq. (2) to each term. Collecting terms involving $\mathrm{v}$ yields

$$
\begin{array}{r}
-2 G(\nu) G(\rho) G(\sigma)+G(\nu) G(\rho+\sigma)+G(\rho) G(\nu+\sigma) \\
+G(\sigma) G(\nu+\rho)-G(\nu+\rho+\sigma)
\end{array}
$$

The corresponding result in $u$ is similar but with negative arguments. These quantities are complex conjugates (c.f. Eq. (3)), so their product equals the square of their magnitude and $\mathrm{Eq} .(\mathrm{C}-27)$ becomes 


$$
\begin{aligned}
W_{\theta}^{C}(f)=\frac{1}{6} \mathcal{F}_{-\infty}^{\infty} d \nu \int_{-\infty}^{\infty} d \rho \int_{-\infty}^{\infty} d \sigma W_{\varphi}(\nu) W_{\varphi}(\rho) W_{\varphi}(\sigma) e^{i 2 \pi(\nu+\rho+\sigma) \tau} \\
\quad \times \mid 2 G(\nu) G(\rho) G(\sigma)-G(\nu) G(\rho+\sigma)-G(\rho) G(\nu+\sigma) \\
-G(\sigma) G(\nu+\rho)+\left.G(\nu+\rho+\sigma)\right|^{2}
\end{aligned}
$$

Applying the Fourier transform to the exponential then yields a delta function $\delta(f-v-\rho-\sigma)$ thereby permitting a simple integration on $v$. Finally, therefore

$$
\begin{aligned}
W_{\theta}^{C}(f)=\frac{1}{6} & \int_{-\infty}^{\infty} d \rho \int_{-\infty}^{\infty} d \sigma W_{\varphi}(f-\rho-\sigma) W_{\varphi}(\rho) W_{\varphi}(\sigma) \\
& \times \mid 2 G(f-\rho-\sigma) G(\rho) G(\sigma)-G(f-\rho-\sigma) G(\rho+\sigma) \\
& -G(\rho) G(f-\rho)-G(\sigma) G(f-\sigma)+\left.G(f)\right|^{2}
\end{aligned}
$$

which is the third of Eqs. (14). 


\section{REFERENCES}

1. Carson, J. R. and T. C. Fry, "Variable Frequency Electric Circuit Theory with Application to the Theory of Frequency Modulation," BSTJ, Vol. 16, No. 4, October 1937, pp. 513-540.

2. Baghdady, E. J., "Theory of Low Distortion Reproduction of FM Signals in Linear Systems, "Trans. IEEE, Vo1. CT-5, No. 3, September 1958, pp. 202-214; "On the Response of a Linear System to an FM Signa 1," Trans. IEEE, Vo1. CT-6, No. 4, December 1959, pp. 387-388.

3. Panter, P. F., Modulation, Noise and Spectral Analysis, McGrawHi11 Book Company, Inc., New York, 1965, Chaps. 8-10.

4. Bloch, A., "Modulation Theory," J. IEE, Vol. 91, Part III, January 1944, pp. 31-42.

5. Van der Pol, B., 'The Fundamental Principles of Frequency Modulation," J. IEE, Vol. 93, Part III, May 1946, pp. 153-158.

6. Stumpers, F.L.H.M., "Distortion of Frequency Modulated Signals in Electrical Networks," Communication News, Vo1. 9, No. 3, Apri1 1948, pp. 82-92.

7. Clavier, A. G., "Application of Fourier Transforms to VariableFrequency Circuit Analysis," Proc. IRE, Vol. 37, No. 11, Novembe: 1949, pp. 1287-1290.

8. Hupert, J. J., "Transient Response of Narrow Band Networks to Angle-Modulated Signals," Proc. N.E.C. , Vol. 18, 1962, pp. 458-468.

9. Hupert, J. J., "Response of Linear Networks to Modulated Waveforms," Proc. IEEE, Vo1. 53, No. 9, September 1965, pp. 12671268 .

10. Weiner, D. D. and B. J. Leon, "On the Quasi-Stationary Response of Linear Time-Invariant Filters to Arbitrary FM Signals," Trans. IEEE, Vo1. CT-11, No. 2, June 1964, Pp. 308-309.

11. Weiner, D. D. and B. J. Leon, "The Quasi-Stationary Response of Linear Systems to Modulated Waveforms," Proc. IEEE, Vo1. 53, No. 6, June 1965, pp. 564-575.

12. Rowe, H. E., "Distortion of Angle-Modulated Waves by Linear Networks," Trans. IEEE, Vo1. CT-9, No. 3, September 1962, pp. 286-290. 
13. Hess, D. T., "Transmission of F.M. Signals through Linear Filters," Proc. N.E.C., Vo1. 18, 1962, pp. 469-476.

14. Frantz, W. J., "The Transmission of a Frequency-Modulated Wave through a Network," Proc. IRE, Vo1. 34, No. 3, March 1946, pp. $114-125$.

15. Gladwin, A. S., "The Distortion of Frequency Modulated Waves by Transmission Networks," Proc. IRE, Vol. 35, No. 12, December 1947, pp. 1436-1445.

16. Zinn, M. K., "Transient Response of a FM Receiver," BSTJ, Vo1. 27, No. 4, October 1948, pp. 715-731.

17. Hupert, J. J., "Normalized Phase and Gain Derivatives as an Aid in Evaluation of FM Distortion," Proc. IRE, Vol. 42, No. 2, February 1954, pp. 438-446.

18. Medhurst, R. G., "Harmonic Distortion of Frequency-Modulated Waves by Linear Networks," Proc. IEE, Vo1. 101, Part III, No. 71, May 1954, pp. 171-181.

19. Turbovich, I. T., "The Effect of Frequency and Amplitude Modulated Oscillations on Linear Systems," Radiotekhnika, Vol. 15, No. 1, 1960, pp. 30-34.

20. Mikheyev, N. G., "Response of an Oscillatory Circuit to PhaseModulated Oscillations for Small Modulation Indices," Radiotekhniki e Elektronika, No. 11, 1964, pp. 80-82.

21. Hupert, J. J., "A Method of Evaluation of the Quasi-Stationary Distortion of FM Signals in Tuned Interstages," Proc. N.E.C., Vo1. 8, 1952, pp. 445-461.

22. Kantor, L. Ya., "Choice of Interstage Coupling Network in Receivers with Frequency Modulation," Elektrosvyaz, No. 4, 1957, pp. 29-32.

23. Kononovich, L. M., "The Bandwidth Required in the I.F. Stage of F.M. Receivers," Elektrosvyaz, No. 11, 1959, pp. 24-33.

24. Rychka, V. L., "The Passage of Frequency Modulated Oscillations Through an Aperiodic Amplifier," Elektrosvyaz, No. 2, 1961, pp. 3-11.

25. Marique, J., "The Response of RLC Resonant Circuits to EMF of Sawtooth Varying Frequency," Proc. IRE, Vo1. 40, No. 8, August 1952, pp. 945-950.

26. Turbovich, I. T., "Dynamic Frequency Characteristics of Selective Systems," Radiotekhnika, Vo1. 12, No. 11, 1957, pp. 39-49. 
27. Go1d, B., "Frequency Transients in Idealized Linear Systems," IRE Conv. Rec., Part 5, 1953, pp. 95-101.

28. McCoy, R. E., "FM Transient Response of Band-Pass Circuits," Proc. IRE, Vo1. 42, No. 3, March 1954, pp. 574-579.

29. Gumowski, I., "Transient Response in FM," Proc. IRE, Vo1. 42, No. 5, May 1954, pp. 819-822; see also M. Nadler and I. Gumowski, "Discussion of 'Transient Response in FM,' by $I$. Gumowski," Proc. IRE, Vo1. 43, No. 6, June 1955, pp. 750-751 and D. A. Linden, "Transient Response in FM," Proc. IRE, Vo1. 45, No. 7, July 1957, pp. 1017-1018.

30. Dennis, F. L. and D. A. Linden, "Computation of FM Transient Response," Proc. IRE, Vo1. 47, No. 9, September 1959, pp. 323-324.

31. Shaft, P. D., "Distortion of Multitone FM Signals Due to Phase Nonlinearity," Trans. IEEE, Vol. SET-9, No. 1, March 1963, pp. 25-35.

32. Prosin, A. V., "Theory of Passing Wideband Signals in EarthSatellite Communications Systems," Radiotekhnika e Elektronika, No. 11, 1963, pp. 1710-1720.

33. Bennett, W. R., H. E. Curtis and S. O. Rice, "Interchannel Interference in FM and PM Systems under Noise Loading Conditions," BSTJ, Vol. 34, No. 3, May 1955, pp. 601-636.

34. Rice, S. D., "Distortion Produced in a Noise Modulated FM Signa1 by Nonlinear Attenuation and Phase Shift," BSTJ, Vol. 36, No. 4, July 1957, pp. 879-889.

35. Bosse, G., "Die Verzerrung frequenz modulierter Schwingungen beim Durchgang durch 1incare Netzwerke," Frequenz, Vo1. 12, October 1958 , pp. 6-14.

36. Medhurst, R. G., "Explicit Form of F.M. Distortion Products with White-Noise Modulation," Proc. IEE, Monograph No. 352E, Vo1. 107, Part c, January 1960, pp. 120-126; see also R. G. Medhurst and J. H. Roberts, "Explicit Form of F.M. Distortion Products with White-Noise Moduiatiuñ-Extension and Correction," Proc. IEE, Monograph No. 352E, Vo1. 107, Part c, September 1960, pp. 36́7-369.

37. Magnusson, R. I., "Intermodulation Noise in Linear F.M. Systems," Proc. IEE, Monograph No. 459E, Vol. 109, Part c, No. 15, March 1962, pp. 32-44.

38. Bedrosian, E. and P. D. Shaft, "Comment on Distortion of Multitone FM Signals Due to Phase Nonlinearity," Trans. IEEE, Vol. SET-10, No. 3, September 1964 , p. 125. 
39. Enloe, L. H. and C. L. Ruthroff, "A Common Error in FM Distortion Theory," Proc. IEEE, Vo1. 51, No. 5, May 1963, p. 846; see also A. S. Gladwin, R. G. Medhurst, L. H. Enloe and C. L. Ruthroff, "A Common Error in FM Distortion Theory, Proc. IEEE, Vo1. 52, No. 2, February 1964, pp. 186-189 and R. I. Magnusson, L. H. Enloe and C. L. Ruthroff, "A Common Error in FM Distortion Theory," Proc. IEEE, Vo1. 52, No. 9, September 1964, pp. 10821084 .

40. Chaffee, J. G., "The Application of Negative Feedback to Frequency Modulation Systems," BSTJ, Vol. 18, No. 3, July 1939, pp. 404-437.

41. Enloe, L. H., "Decreasing the Threshold in FM by Frequency Feedback," Proc. IRE, Vo1. 50, No. 1, January 1962, pp. 18-30.

42. Ruthroff, C. L. and W. F. Bodtmann, "Design and Performance of a Broad-Band FM Demodulator with Frequency Compression," Proc. IRE, Vo1. 50, No. 12, December 1962, pp. 2436-2445.

43. Giger, A. J. and J. G. Chaffee, "The FM Demodulator with Negative Feedback," BSTJ, Vol. 42, No. 4, Part 1, July 1963, pp. 1109-1135.

44. Hille, E. and R. S. Phillips, Functional Analysis and Semi-Groups, rev. ed., American Mathematical Society Colloquium Publications, Vo1. XXXI, American Mathematical Society, Providence, Rhode Island, 1957, Chaps. 3 and 26.

45. Rice, S. O., "Mathematical Analysis of Random Noise," BSTJ, Vol. 23, No. 3, July 1944, pp. 282-332 and Vol. 24, No. 1, January 1945, pp. 46-156; see also N. Wax (ed.), Selected Papers on Noise and Stochastic Processes, Dover Publications, New York, 1945 .

46. Bromwich, T. J. I'a., An Introduction to the Theory of Infinite Series, 2d ed. rev., MacMillan and Company, London, 1942, p. 499 .

47. Henderson, K. W. and W. H. Kautz, "Transient Responses of Conventional Filters," Trans. IRE, Vo1. CT-5, No. 4, December 1958, pp. 333-347; see also G. A. Beck, "Comments on'Transient Responses of Conventional Filters," Trans. IRE, Vol. CT-8, No. 2, June 1961, pp. 166-167 and E. van Vollenhoven and H. A. Reuver, "Comments on 'Transient Responses of Conventional Filıers," Trans. IRE, Vo1. CT-8, No. 4, December 1961, p. 500.

48. Wang, M. C. and G. E. Uhlenbeck, "On the Theory of the Brownian Motion II," Rev. Mod. Phys., Vol. 17, Nos. 2 and 3, April-July 1945, pp. 323-342; see also N. Wax (ed.), Selected Papers on Noise and Stochastic Processes, Dover Publications, New York, 1945 . 